\title{
Time-Resolved Measurements and Master Equation Modelling of the Unimolecular Decomposition of $\mathrm{CH} 3 \mathrm{OCH} 2$
}

\section{Eskola, Arrke J.}

2020-07

Eskola , A J , Blitz , M A , Pilling, M J , Seakins , P W \& Shannon , R J 2020 , '

Time-Resolved Measurements and Master Equation Modelling of the Unimolecular

Decomposition of $\mathrm{CH} 3 \mathrm{OCH} 2$ ' , Zeitschrift für physikalische Chemie , vol. 234 , no. 7-9 , pp.

1233-1250 . https://doi.org/10.1515/zpch-2020-0007

http://hdl.handle.net/10138/328821

https://doi.org/10.1515/zpch-2020-0007

acceptedVersion

Downloaded from Helda, University of Helsinki institutional repository.

This is an electronic reprint of the original article.

This reprint may differ from the original in pagination and typographic detail.

Please cite the original version. 
Time-Resolved Measurements and Master Equation Modelling of the Unimolecular Decomposition of $\mathrm{CH}_{3} \mathrm{OCH}_{2}$
A.J Eskola, ${ }^{1,2}$ M.A Blitz, ${ }^{1,3}$ M.J. Pilling, ${ }^{1}$ P.W Seakins ${ }^{1}$ and R.J. Shannon ${ }^{1}$

${ }^{1}$ School of Chemistry, University of Leeds, Leeds, LS2 9JT, UK.

${ }^{2}$ Department of Chemistry, University of Helsinki, P.O. Box 55 (A.I. Virtasen aukio 1), FI00014 Helsinki, Finland

${ }^{3}$ National Centre for Atmospheric Science, University of Leeds, Leeds, LS2 9JT, UK 


\begin{abstract}
The rate coefficient for the unimolecular decomposition of $\mathrm{CH}_{3} \mathrm{OCH}_{2}, k_{1}$, has been measured in time-resolved experiments by monitoring the $\mathrm{HCHO}$ product. $\mathrm{CH}_{3} \mathrm{OCH}_{2}$ was rapidly and cleanly generated by $248 \mathrm{~nm}$ excimer photolysis of oxalyl chloride, $(\mathrm{ClCO})_{2}$, in an excess of $\mathrm{CH}_{3} \mathrm{OCH}_{3}$, and an excimer pumped dye laser tuned to $353.16 \mathrm{~nm}$ was used to probe $\mathrm{HCHO}$ via laser induced fluorescence. $k_{1}(T, p)$ was measured over the ranges: $573-673 \mathrm{~K}$ and $0.1-4.3$ $\times 10^{18}$ molecule $\mathrm{cm}^{-3}$ with a helium bath gas. In addition, some experiments were carried out with nitrogen as the bath gas.

Ab initio calculations on $\mathrm{CH}_{3} \mathrm{OCH}_{2}$ decomposition were carried out and a transition-state for decomposition to $\mathrm{CH}_{3}$ and $\mathrm{H}_{2} \mathrm{CO}$ was identified. This information was used in a master equation rate calculation, using the MESMER code, where the zero-point-energy corrected barrier to reaction, $\Delta E_{0,1}$, and the energy transfer parameters, $\left\langle\Delta E_{\text {down }}\right\rangle \times T^{\mathrm{n}}$, were the adjusted parameters to best fit the experimental data, with helium as the buffer gas. The data were combined with earlier measurements by Loucks and Laidler (Can J. Chem. 1967, 45, 2767), with dimethyl ether as the third body, reinterpreted using current literature for the rate coefficient for recombination of $\mathrm{CH}_{3} \mathrm{OCH}_{2}$. This analysis returned $\Delta E_{0,1}=(112.3 \pm 0.6) \mathrm{kJ}$ $\mathrm{mol}^{-1}$, and leads to $k_{1}^{\infty}(T)=2.9 \times 10^{12}(T / 300)^{2.5} \exp \left(-106.8 \mathrm{~kJ} \mathrm{~mol}^{-1} / \mathrm{R} T\right)$. Using this model, limited experiments with nitrogen as the bath gas allowed $N_{2}$ energy transfer parameters to be identified and then further MESMER simulations were carried out, where $\mathrm{N}_{2}$ was the buffer gas, to generate $k_{1}(T, p)$ over a wide range of conditions: $300-1000 \mathrm{~K}$ and $\mathrm{N}_{2}=10^{12}-10^{25}$ molecule $\mathrm{cm}^{-3}$. The resulting $k_{1}(T, p)$ has been parameterized using a Troe-expression, so that they can be readily be incorporated into combustion models. In addition, $k_{1}(T, p)$ has been parametrized using PLOG for the buffer gases, $\mathrm{He}, \mathrm{CH}_{3} \mathrm{OCH}_{3}$ and $\mathrm{N}_{2}$.
\end{abstract}




\section{Introduction}

The low temperature $(500-1000 \mathrm{~K})$ combustion of organic compounds involves the initial generation of a radical, $\mathrm{R}$, usually by reaction of the parent molecule with $\mathrm{OH}$. $\mathrm{R}$ reacts with $\mathrm{O}_{2}$ to form a peroxy radical and, following isomerization and further reaction with $\mathrm{O}_{2}$ chain branching reactions can occur, leading to ignition.[1,2] The mechanism is generally termed autoignition. The overall rate of reaction increases with temperature up to where the negative temperature coefficient (NTC) range is encountered, when the rate decreases. The NTC results from the shift in the $\mathrm{R}+\mathrm{O}_{2}=\mathrm{RO}_{2}$ equilibrium and, particularly for ethers, dissociation of the radical, $\mathrm{R}$, competing with its reaction with $\mathrm{O}_{2}$. This suppresses branching, because the dissociation products promote autoignition less effectively. Rate coefficients for radical dissociation are essential components of autoignition mechanisms.

Dimethyl ether (DME) is a potential alternative fuel for combustion engines.[3,4] The propagating radical, methoxymethyl, $\mathrm{CH}_{3} \mathrm{OCH}_{2}$, is formed from the $\mathrm{OH}+\mathrm{DME}$ reaction and dissociates via reaction (R1)

$$
\mathrm{CH}_{3} \mathrm{OCH}_{2} \quad \rightarrow \quad \mathrm{CH}_{3}+\mathrm{HCHO}
$$

forming the methyl radical which does not undergo low temperature autoignition chemistry. While the kinetics of $\mathrm{OH}+\mathrm{DME}$ have been widely studied,[5-7]reaction (R1) is much less well characterised.

Loucks and Laidler [8] measured $k_{1}$ relative to the rate coefficient for $\mathrm{CH}_{3}+\mathrm{CH}_{3}$, using mercury photosensitisation coupled with end product analysis by gas chromatography. [8] They used a Lindemann Hinshelwood mechanism to analyse their data, reporting the high and low pressure limiting rate coefficient $k_{1}$. Their measurements spanned temperatures of $473-573$ $\mathrm{K}$ and pressures of $4 \times 10^{-3}-0.8$ bar; the bath gas was DME. For the high pressure limiting rate coefficient they obtained $k_{1}^{\infty}=1.6 \times 10^{13} \exp \left(-106 \mathrm{kJmol}^{-1} / R T\right) \mathrm{s}^{-1}$. Sehested et al. [9] used pulse radiolysis coupled with time-resolved uv absorption to study a range of reactions of $\mathrm{CH}_{3} \mathrm{OCH}_{2}$ in $\mathrm{SF}_{6}$ at 18.3 bar and over the temperature range $296-666 \mathrm{~K}$. Their results are consistent with the extrapolation of $k_{1}^{\infty}$ from Loucks and Laidler.

Additionally, methoxymethyl decomposition has been investigated using high level electronic structure calculations. Li et al.[10] obtained $k_{1}^{\infty}=4.45 \times 10^{14}(T / \mathrm{K})^{-0.22} \exp (-13700 \mathrm{~K} / T) \mathrm{s}^{-1}$ which 
lies within a factor of $\sim 2$ of the results of Loucks and Laidler over the temperature range 400 - 800 K. Gao et al.[11] incorporated multiple structure effects into their calculations to account for torsional and anharmonic effects for the low frequency modes. They obtained $k_{1}^{\infty}=1.88 \times 10^{12}(T / 300 \mathrm{~K})^{1.05} \exp (-11062 \mathrm{~K} / T) \mathrm{s}^{-1}$, over the temperature range $450-800 \mathrm{~K}$, which is a factor of $\sim 10$ to $\sim 3$ greater than the expression of Loucks and Laidler. Gao et al. also used an RRKM analysis to obtain the pressure dependence and the limiting rate coefficients $k_{1}^{\infty}$ and $k_{1}{ }^{0}$.

Curran et al.[12,13] developed a detailed mechanism for DME pyrolysis and oxidation. They incorporated the Loucks and Laidler[8] high pressure limit value of $k_{1}$ but did not include any pressure dependence. They tested the mechanism against the jet stirred reactor studies of Dagaut et al.[14] and found a high negative sensitivity for $k_{1}$ at $850 \mathrm{~K}$ and 10 atm, but a lower sensitivity at $950 \mathrm{~K}$. Curran et al. subsequently carried out studies in a variable pressure flow reactor (12 - $18 \mathrm{~atm}, 550-850 \mathrm{~K})$ in a nitrogen diluent.[15] Their analysis showed a high negative sensitivity to $k_{1}$ at $750 \mathrm{~K}$, but low sensitivity at lower temperatures.

This paper reports the first direct measurements of $k_{1}$, over the temperature range $573-673$ $\mathrm{K}$ in a helium diluent, using pulsed laser photolysis to indirectly, but rapidly, generate $\mathrm{CH}_{3} \mathrm{OCH}_{2}$ with detection of the $\mathrm{HCHO}$ product with laser induced fluorescence. The results were compared with those from a master equation model, using the code MESMER, based on electronic structure calculations of the reactant and transition state. The best fit was determined by minimising $\chi^{2}$ using the barrier height and the temperature dependent energy transfer parameter, $\left\langle\Delta E_{\text {down }}\right\rangle$, as variable parameters. Parameterized forms are given for $k_{1}([\mathrm{M}], T)$. 


\section{Methods}

\subsection{Experimental}

Time-resolved studies were conducted in a pulsed photolysis-laser induced fluorescence (LIF) apparatus that has been described in previous publication.[16] Briefly, a stainless steel sixway cross reactor provided the optical axes for the lasers to initiate reaction and for radical detection, where the temperature was set via a central heating block ( $T=573-673 \mathrm{~K}$ ). The gases were delivered to the reactor via calibrated mass flow controllers, either directly from a cylinder $(\mathrm{He})$ or via bulbs containing the reagents $\left(\mathrm{CH}_{3} \mathrm{OCH}_{3}\right.$ and $\left.(\mathrm{ClCO})_{2}\right)$ diluted with the buffer gas. The pressure of the gases was controlled via a needle valve on the exhaust line and monitored by pressure gauges (MKS) attached to the reactor. Photolysis was initiated by a KrF excimer laser (Lambda Physik, LPX105) that typically gave $40 \mathrm{~mJ} / \mathrm{pulse} / \mathrm{cm}^{2}$ output at $248 \mathrm{~nm}$, and generated $\mathrm{Cl}$ by photolysis of oxalyl chloride, $(\mathrm{ClCO})_{2}$.[17] The probe laser was a $308 \mathrm{~nm}$ excimer (Lambda Physik LPX105) pumped dye laser (Lambda Physik 3002) operating on the dye DMQ to produce light at $353.16 \mathrm{~nm}$.[18] The photolysis and probe light interact only in the centre of the reactor and together define the reaction zone. A thermocouple was located close to the reaction zone. The detection port was perpendicular to the plane defined by the two lasers and the laser induced fluorescence was imaged via a lens and passed through a filter (Perspex) onto the photomultiplier tube (PMT) detector. Formaldehyde, $\mathrm{HCHO}$, has a well-known fluorescence spectrum [19] lying above $350 \mathrm{~nm}$. The fluorescence is short lived, $<50 \mathrm{~ns}$, even at low pressures and is not significantly quenched even at a few hundred Torr of nitrogen. The fluorescence intensity peaks at wavelengths greater than 400 $\mathrm{nm}$, so a Perspex filter (transmits $>385 \mathrm{~nm}$ ) removes laser scatter without significantly decreasing the emission intensity. Further details can be found in Gravestock et al.[18]

A delay generator (SRS, DG 535) was used to delay the probe laser relative to the photolysis laser and the delay time was scanned in order to build up the $\mathrm{HCHO}$ time trace, see Figure 1 for an example. The fluorescence detected via the PMT was passed to an oscilloscope (LeCroy, LT262), where it was displayed and integrated before being transferred and stored on a computer. The delay generator and oscilloscope were controlled via LabVIEW and the decay trace was stored for analysis. 
Helium (BOC, CP) was used directly from the cylinder. (CICO) ${ }_{2}$ (Sigma Aldrich) and DME (Sigma Aldrich) were degassed before dilution in glass bulbs. The total flows were typically 10 SCCM / Torr, which meant the reaction cell was replenished in $\sim 5$ seconds (volume of reaction cell $\sim 600 \mathrm{~cm}^{3}$ ), independent of the total pressure.

\subsection{Theory}

The analysis relies on master equation simulations with the MESMER software,[20] which requires characterisation of the important species (reactant and transition state) using electronic structure theory. Geometry optimisations were performed for all species at the M06-2X/ 6-31+G** level of theory using the Gaussian 09 software.[21] The energetics for the reaction were refined through single point energy calculations at optimised geometries using the ROHF-UCCSD(T)-F12/aug-cc-pVTZ formalism employed in the Molpro software.[22] As found by Gao et al., the T1 diagnostics for both the well $\left(\mathrm{CH}_{3} \mathrm{OCH}\right)$ and TS were found to be below the commonly cited threshold of 0.045 , indicating that a single reference method should be appropriate for the current system. To correctly characterise hindered internal rotation of the $\mathrm{CH}_{3}$ and $\mathrm{CH}_{2}$ groups, relaxed torsional scans were performed at the M06-2X/ $6-31+G^{* *}$ level of theory for use with the $Q M$ hindered rotor methodology used in MESMER.[23] The internal rotor modes were projected from the Hessian and the energy levels calculated based on the numerical potentials.

The calculated molecular parameters were incorporated in the master equation code MESMER and the input file is given in the online supplementary information. A harmonic oscillator - rigid rotor approximation was used for all modes, other than the internal rotors, in determining the ro-vibrational densities of states of each species. The $\mathrm{CH}_{3}$ and $\mathrm{CH}_{2}$ torsional motions were projected out of the vibrational Hessian as described by Sharma et al.[24] Energy transfer [25] was treated with an exponential down model and $\left\langle\Delta E_{\text {down }}\right\rangle$, (the average energy transferred in a downward direction upon collision between $\mathrm{CH}_{3} \mathrm{OCH}_{2}$ and either a $\mathrm{He}$, $\mathrm{N}_{2}$, or $\mathrm{CH}_{3} \mathrm{OCH}_{3}$ bath gas molecule) was used as a fitting parameter in the master equation simulations. Collision rates between $\mathrm{CH}_{3} \mathrm{OCH}_{2}$ and the bath were determined using a LennardJones model with the Lennard-Jones parameters $\varepsilon$ and $\sigma$ determined using the Joback method [26] as implemented in the RMG software suite [27] (see Supplementary Information). 
MESMER uses the Levenberg-Marquardt method of fitting, minimising $\chi^{2}$ in a comparison of calculated and experimental rate coefficients. The only variable parameters used were the difference in zero point corrected energies between the TS and reactant, $\Delta E_{0,1}$, and $\left\langle\Delta E_{\text {down }}\right\rangle$, which was given a $T^{n}$ dependence. The fixed input parameters are given in the SI. MESMER uses matrix techniques and the present analysis is essentially based on the determination in MESMER of the numerically smallest eigenvalue, the single, chemically significant eigenvalue (CSE) for the reaction system. This eigenvalue is well separated from the numerically larger values that relate to internal energy relaxation, so that the use of the CSE to determine $k_{1}$ is valid.

\section{Results}

Experiments were carried out using helium as the buffer gas, and the pressures and temperatures were varied over the ranges $7.5-280$ Torr and $573-673 \mathrm{~K}$. The $\mathrm{CH}_{3} \mathrm{OCH}_{2}$ radical was generated via the reaction between chlorine atoms and dimethyl ether:

$$
\mathrm{Cl}+\mathrm{CH}_{3} \mathrm{OCH}_{3} \rightarrow \mathrm{CH}_{3} \mathrm{OCH}_{2}+\mathrm{HCl}
$$

This reaction is fast, with a rate coefficient $>10^{-10} \mathrm{~cm}^{3}$ molecule $\mathrm{s}^{-1},[28]$ and exclusively generates $\mathrm{CH}_{3} \mathrm{OCH}_{2}$. The precursor $\mathrm{BrCH}_{2} \mathrm{OCH}_{3}$ was previously used to generate $\mathrm{CH}_{3} \mathrm{OCH}_{2}$ photolytically,[29] but the yields of $\mathrm{CH}_{3} \mathrm{OCH}_{2}$, and of other product channels, were not established. As many reactions can generate formaldehyde, reaction R2 better guarantees that the observed $\mathrm{HCHO}$ is formed exclusively via R1. In all cases, the conditions were arranged such that the rate of $\mathrm{R} 2$ was much greater than that of $\mathrm{R} 1\left(k_{2}^{\prime} " k_{1}{ }^{\prime}\right)$. The exothermicity of reaction $\mathrm{R} 2\left(-28 \mathrm{~kJ} \mathrm{~mol}^{-1}\right)$ is much less than the energy required for $\mathrm{CH}_{3} \mathrm{OCH}_{2}$ dissociation, R1, so prompt $\mathrm{HCHO}$ formation is not possible.

The formation of $\mathrm{HCHO}$ in the system is via reactions $\mathrm{R} 1$ and $\mathrm{R} 2$, where $k_{2}\left[\mathrm{CH}_{3} \mathrm{OCH}_{3}\right] \gg k_{1}$, and $\mathrm{HCHO}$ is lost via diffusion to outside the volume, defined by the excimer photolysis beam, cross-section $2.5 \times 1.0 \mathrm{~cm}^{2}$ :

$$
\mathrm{HCHO} \stackrel{\text { diffusion, } k_{d}}{\longrightarrow} \text { loss }
$$

The kinetic solution to this scheme is:

$$
[\mathrm{HCHO}]=[\mathrm{Cl}]_{0}\left\{\left(\exp \left(-k_{d} t\right)-\exp \left(-k_{1} t\right)\right\}+b\right.
$$


where $[\mathrm{Cl}]_{0}$ is the concentration of $\mathrm{Cl}$ generated from the $248 \mathrm{~nm}$ photolysis of oxalyl chloride: [30]

$$
(\mathrm{ClCO})_{2} \stackrel{248 \mathrm{~nm}}{\longrightarrow} \quad 2 \mathrm{Cl}+2 \mathrm{CO} \quad \mathrm{P} 1
$$

At $248 \mathrm{~nm}$, initially a fraction of the photolysis produces $\mathrm{ClCO}$ but this decomposes to $\mathrm{Cl}$ and $\mathrm{CO}$ rapidly at the temperatures of the present experiments.[30] $\mathrm{E} 1$ assumes $\mathrm{CH}_{3} \mathrm{OCH}_{2}$ loss is exclusively via R1 and $k_{d}$ is diffusional loss of $\mathrm{HCHO}$. The red line in Figure 1 is the best fit of equation $\mathrm{E} 1$ to the data. The $b$ parameter takes into account background $\mathrm{HCHO}$ in the reaction cell.

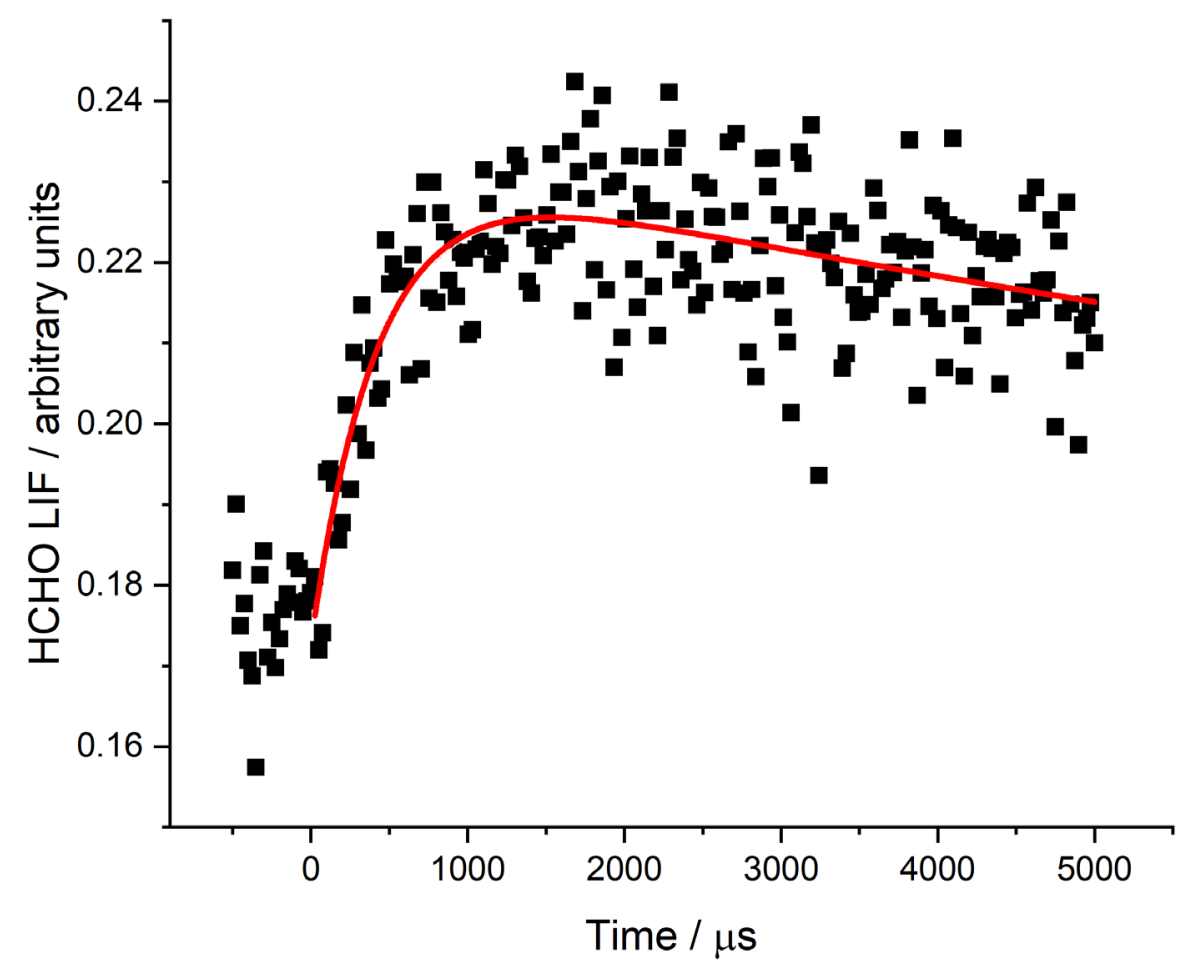

Figure 1. An example of the $\mathrm{HCHO}$ data at $622 \mathrm{~K}$ using helium as the buffer gas. The total pressure was 53.2 Torr, $(\mathrm{COCl})_{2}$ and $\mathrm{CH}_{3} \mathrm{OCH}_{3}$ were equal to $7.37 \times 10^{14}$ and $1.39 \times 10^{15}$ molecule $\mathrm{cm}^{-3}$, respectively. The laser repetition rate was $5 \mathrm{~Hz}$ while the cell residence time was $5 \mathrm{~s}$, so that there is a background of $\mathrm{HCHO}$ which can be determined from the signal before zero time (see text). 
As expected, the laser induced fluorescence (LIF) signal from $\mathrm{HCHO}$ was degraded as the temperature was increased. For this reason $\left[\mathrm{CH}_{3} \mathrm{OCH}_{2}\right]\left(\sim 10^{13}\right.$ molecule $\left.\mathrm{cm}^{-3}\right)$ was higher than would normally be used in a LFP/LIF experiments, which implies there was removal of $\mathrm{CH}_{3} \mathrm{OCH}_{2}$ via the self-reaction:

$$
\mathrm{CH}_{3} \mathrm{OCH}_{2}+\mathrm{CH}_{3} \mathrm{OCH}_{2} \rightarrow \text { products R4 }
$$

especially at $573 \mathrm{~K}$, where dissociation is slower. The rate coefficient for reaction $\mathrm{R} 4$ has been measured at room temperature [9] and above,[31] and it is $\sim 2 \times 10^{-11} \mathrm{~cm}^{3}$ molecule ${ }^{-1} \mathrm{~s}^{-1}$ at the elevated temperatures of the present experiments, so that $k_{3}\left[\mathrm{CH}_{3} \mathrm{OCH}_{2}\right] \sim 200 \mathrm{~s}^{-1}$. The impact of the self-reaction, R4, on the observed kinetics is greatest at the lowest temperature, $573 \mathrm{~K}$, where dissociation is slowest and was assessed by analysing traces numerically, including $\mathrm{R} 3$ as a second order reaction in $\mathrm{CH}_{3} \mathrm{OCH}_{2}$ with $[\mathrm{CH} 3 \mathrm{OCH} 2]=1 \times 10^{13}$ molecule $\mathrm{cm}^{-}$ 3 . It was observed that the fitted value returned for $k_{1}$ was lowered by $\sim 200 \mathrm{~s}^{-1}$ compared with the analysis when R4 was omitted. This $200 \mathrm{~s}^{-1}$ was subtracted from the observed decay constant to determine $\mathrm{k}_{1}$ and an additional $200 \mathrm{~s}^{-1}$ error was propagated into all the $\mathrm{k}_{1}$ errors. This results in a large total error at $573 \mathrm{~K}$, but much reduced errors at the higher temperatures, where $k_{1} \sim 10^{3}-2 \times 10^{4} \mathrm{~s}^{-1}$. These total uncertainties are shown in Figs. 2 and 4. The net result is that the $573 \mathrm{~K}$ data contribute little to the MESMER analysis.

The detected $\mathrm{HCHO}$ is a stable molecule, therefore it was only removed when pumped out of the reaction cell. Typically, the cell is fully replenished every 5 seconds. However, the photolysis light only illuminated a fraction of the cell volume, therefore the laser repetition does not need to be as slow as $(1 / 5) \mathrm{s}^{-1}$. In fact, the only requirement is that $\mathrm{HCHO}$ from reaction $\mathrm{R} 1$ is visible above the background $\mathrm{HCHO}$ obtained from previous laser samples, and this requirement was met when the repetition rate was $\leq 5 \mathrm{~Hz}$. In Figure 1 the data were collected at $5 \mathrm{~Hz}$, where it can be seen that there is significant residual [HCHO] before time zero.

The experimental results with helium as bath gas are given in Table 1. These results (16 points) were combined with those of Loucks and Laidler (66 points) in a global master equation analysis. Their full dataset was only presented graphically and this was first digitised which potentially introduces some systematic error. Their values of $k_{1}$ were determined by end 
product analysis, comparing the products of dissociation with those of recombination of $\mathrm{CH}_{3} \mathrm{OCH}_{2}$. The product ratio is equal to $k_{1} /\left(k_{4}\right)^{1 / 2}$. The high pressure limiting rate coefficient for $\mathrm{CH}_{3}+\mathrm{CH}_{3}$ from measurements of Shepp [32] $\left(3.7 \times 10^{-11} \mathrm{~cm}^{3}-\right.$ molecule $\left.{ }^{-1} \mathrm{~s}^{-1}\right)$, was used for $k_{4}$ in their analysis. For the present study, these data were recalculated using the rate coefficient for $\mathrm{CH}_{3} \mathrm{OCH}_{2}+\mathrm{CH}_{3} \mathrm{OCH}_{2}$ determined by Maricq et al.[31] The resulting rate coefficients are given in the SI and are each assigned a 20\% error.

The master equation analysis, via MESMER, used the zero-point energy corrected barrier with respect to $\mathrm{CH}_{3} \mathrm{OCH}_{2}, \Delta E_{0,1}$, and the temperature dependent energy transfer parameters for helium and $\mathrm{DME},\left\langle\Delta E_{\text {down }}>\times T^{\mathrm{n}}\right.$, to calculate $k_{1}$ and compare with the experimental values. MESMER allows fitting to different values of $\left\langle\Delta E_{\text {down }}>\right.$ for He and DME in the same global fit. The best fit values of $k_{1}$ (calc) are given in Table 1 . The best fit parameters, based on minimizing the weighted $\chi^{2}\left(k_{1}(\mathrm{calc})-k_{1}(\mathrm{expt})\right)^{2}$, are given in Table 2 , where the weight uses the $k_{1}$ errors given in Table 1. The value of $\chi^{2}$ is 72.5, which implies that the errors in Table 2 are close to 1б. The fits are shown in Figure 2, where lines though the data are from the MESMER fit. The SI provides a comparison of the data from Laidler and Loucks with the results from the best fit model.

The results when $\mathrm{N}_{2}$ was the bath gas were incompatible with the present results at higher pressures. This problem arises because of incomplete heat transfer under higher pressure conditions, so that the temperatures recorded on the thermocouples did not accurately record the temperature of the probed reaction zone. The problem was identified by using chemical thermometry, by measuring the rate constant for $\mathrm{OH}+\mathrm{CH}_{4}$ under the experimental conditions. This method showed that there were no problems with $\mathrm{He}$ as the bath gas, under the conditions studied, because of its higher thermal conductivity. This problem with using nitrogen as the buffer gas has been identified when using a higher pressure system [33] and will be addressed in a future publication.

In our previous publication on $\mathrm{CH}_{3} \mathrm{OCH}_{2}+\mathrm{O}_{2}$, [34] energy transfer parameters when $\mathrm{N}_{2}$ was the buffer gas were determined, $\left\langle\Delta E_{\text {down }}>=523 \pm 53 \mathrm{~cm}^{-1}\right.$ and $n=0.01 \pm 0.17,(T / 298)^{n}$. MESMER simulations were made to generate rate coefficients with $\mathrm{N}_{2}$ as the buffer gas, using these energy transfer values, coupled with the best fit value for $\Delta E_{0,1}$ obtained from fitting 
the He data. The resulting $k_{1}(T, p)$ were parameterized using a Troe expression, and the result is given in Table 2. PLOG parameters are included in the SI.

Table 1. Experimental values obtained for $k_{1}$ with helium as the buffer gas. The error is equal to $\pm 20 \% k_{1}$ propagated with an additional error from the contribution from radical - radical reaction with an effective first order rate coefficient of $200 \mathrm{~s}^{-1}$. This contribution accounts for the high uncertainties at $573 \mathrm{~K}$. Also tabulated are the calculated $k_{1}$ from the MESMER analysis.

\begin{tabular}{|c|c|c|c|c|}
\hline $10^{-17}[\mathrm{He}] / \mathrm{cm}^{-3}$ & $T / \mathrm{K}$ & $\left(k_{1} / \mathrm{s}^{-1}\right)($ expt $)$ & error $/ \mathrm{s}^{-1}$ & $\left(k_{1} / \mathrm{s}^{-1}\right)(\mathrm{calc})$ \\
\hline 1.23 & 573 & 38 & 304 & 124 \\
\hline 9.24 & 573 & 791 & 360 & 392 \\
\hline 21.0 & 573 & 767 & 360 & 584 \\
\hline 18.5 & 573 & 396 & 323 & 551 \\
\hline 1.14 & 622 & 950 & 380 & 560 \\
\hline 1.14 & 622 & 2750 & 670 & 560 \\
\hline 8.50 & 622 & 1950 & 520 & 1943 \\
\hline 19.3 & 622 & 3350 & 760 & 3015 \\
\hline 17.0 & 622 & 4150 & 920 & 2824 \\
\hline 43.4 & 622 & 5970 & 1240 & 4438 \\
\hline 1.05 & 673 & 1770 & 500 & 2011 \\
\hline 1.05 & 673 & 2520 & 617 & 2011 \\
\hline 7.87 & 673 & 7350 & 1529 & 7590 \\
\hline 7.87 & 673 & 4780 & 1044 & 7590 \\
\hline 15.8 & 673 & 13700 & 2816 & 11400 \\
\hline 40.1 & 673 & 23300 & 4709 & 18786 \\
\hline
\end{tabular}




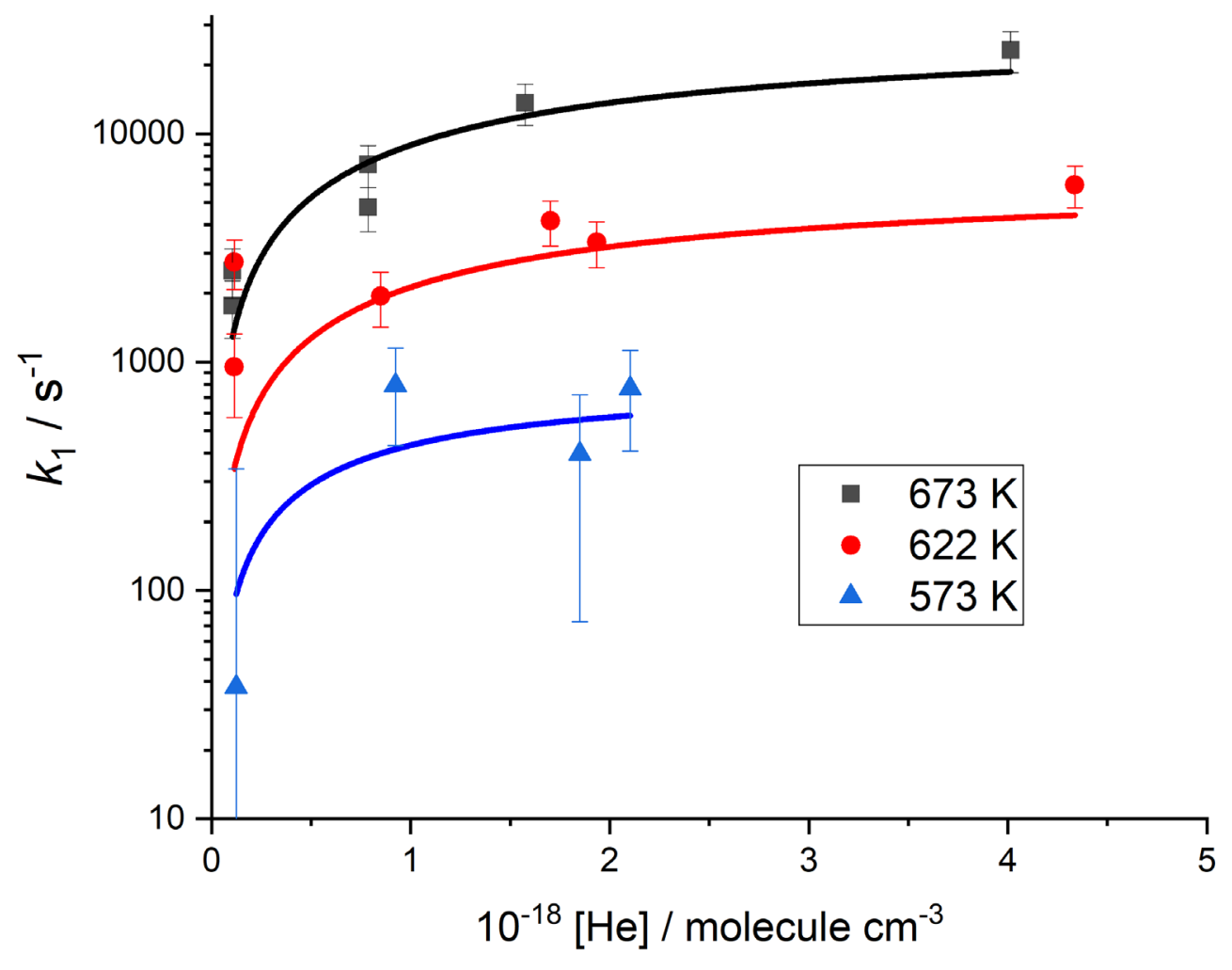

Figure 2. $k_{1}(T)$ unimolecular decomposition rate coefficient data obtained when helium was the buffer gas. $k_{1}$ at a given temperature is behaving in a consistent way as a function of total pressure as evidenced by master equation fitting to the data, the lines through the data. 
Table 2. MESMER fitted parameters for $k_{1}$ together with literature data, and the Troe fitting parameters to the MESMER simulations using $\mathrm{N}_{2}$ as the buffer gas. The high pressure limit is given in the form: $k_{1}(T)=A(T / 300)^{n} \exp \left(-E a_{1} / R T\right) \mathrm{s}^{-1}$. Errors are $1 \sigma$.

\begin{tabular}{|c|c|c|c|c|c|}
\hline & This work & Li et al. & Gao et al..$^{(c)}$ & $\begin{array}{c}\text { Loucks } \\
\text { and } \\
\text { Laidler (e) }\end{array}$ & $\begin{array}{c}\text { Troe } \\
\text { parameters } \\
\left(\mathrm{N}_{2}\right)^{(\mathrm{f})}\end{array}$ \\
\hline$\Delta E_{0,1} / \mathrm{kJ} \mathrm{mol}^{-1}$ & $\begin{array}{c}112.5 \pm 0.6^{(a)} \\
110.8^{(b)}\end{array}$ & & $\begin{array}{l}110.6^{\text {(c) }} \\
111.6^{\text {(d) }}\end{array}$ & & \\
\hline $\begin{array}{l}<\Delta E_{\text {down, } \mathrm{He}}>x \\
(T / 298)^{n} / \mathrm{cm}^{-1}\end{array}$ & $\begin{array}{l}195 \pm 320 \\
0.77 \pm 2.0\end{array}$ & & & & \\
\hline $\begin{array}{l}<\Delta E_{\text {down,DME }}>\times \\
(T / 521)^{n} / \mathrm{cm}^{-1}\end{array}$ & $\begin{array}{c}871 \pm 611 \\
-0.87 \pm 1.0\end{array}$ & & & & \\
\hline$A_{1}^{\infty} / \mathrm{s}^{-1}$ & $2.89 \times 10^{12}$ & $\begin{array}{c}1.27 \times \\
10^{14}\end{array}$ & $2.08 \times 10^{12}$ & $1.6 \times 10^{13}$ & $2.19 \times 10^{12}$ \\
\hline$n$ & 2.51 & -0.22 & 1.002 & 0 & 2.77 \\
\hline$E a_{1}{ }^{\infty} / \mathrm{kJ} \mathrm{mol}^{-1}$ & 106.8 & 113.90 & 92.27 & 106.7 & 106.22 \\
\hline$A_{1}{ }^{0} / \mathrm{s}^{-1}$ & & & $4.1 \times 10^{-8}$ & $4.6 \times 10^{-8}$ & 729 \\
\hline $\mathrm{m}$ & & & 0.053 & 0 & -8.53 \\
\hline$E a_{1}{ }^{0} / \mathrm{kJ} \mathrm{mol}^{-1}$ & & & 75.4 & 75.7 & 115.63 \\
\hline$b$ & & & & & 0.469 \\
\hline$x_{0}$ & & & & & 1.30 \\
\hline$F_{\text {cent }} \mathrm{A}$ & & & & & $3.04 \times 10^{-4(f)}$ \\
\hline
\end{tabular}

(a) Results from the MESMER fitting to the experimental results provided here, with $\mathrm{He}$ as diluent and from the data of Loucks and Laidler, [8] in DME, rescaled using the data of Maricq et al. for $k_{4 .}{ }^{(b)}$ From Leeds ab initio calculations (ROHF-UCCSDT-f12/aug-cc-pVTZ). (c) From a G4 calculation. (d) From a CCSD(T)/aug-cc-PVTZ//M062X/MG3S calculation. (e) The lowpressure limit is for dimethyl ether as the buffer gas. These are the original results given by Loucks and Laidler; the rate coefficients have not been scaled to incorporate the data of Maricq et al. for $k_{4}{ }^{(f)} F_{c e n t} B$ and $F_{c e n t} C$ are equal to zero. 


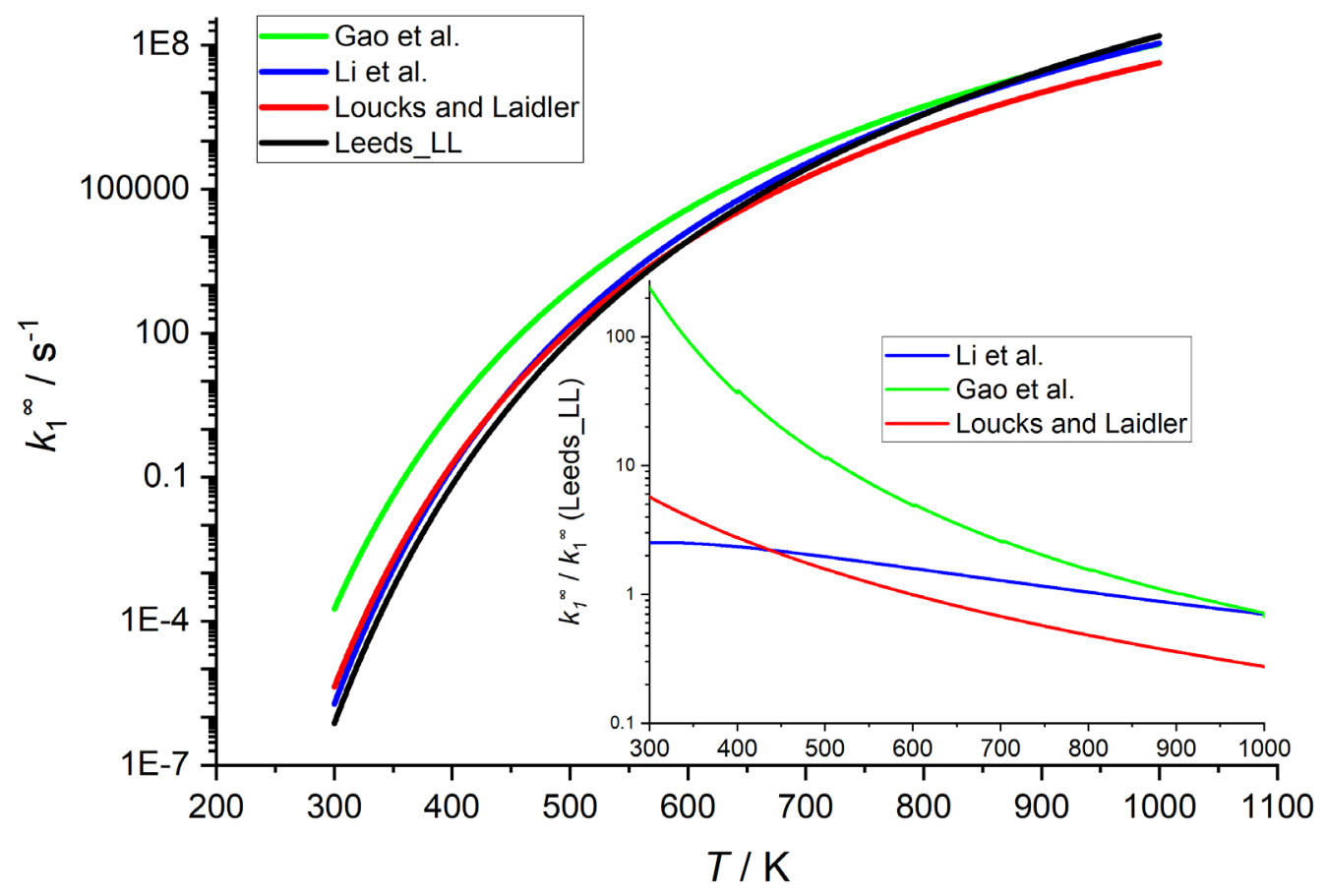

Figure $3 k_{1}^{\infty}(T)$ from the literature and this study. The Loucks and Laidler line refers to the parameters from their original study and Leeds_LL is the result from this study, which includes the Loucks and Laidler data, with the data rescaled using the $k_{4}$ values of Maricq et al. The inset shows $k_{1}^{\infty} / k_{1}^{\infty}$ (Leeds_LL).

\section{Discussion}

The first study on the thermal decomposition of $\mathrm{CH}_{3} \mathrm{OCH}_{2}$ was the relative rate study by Loucks and Laidler,[8] where the decomposition was carried out between $473-573 \mathrm{~K}$ with dimethyl ether as the buffer gas. A Lindemann-Hinshelwood expression was used to extrapolate the data to the low and high pressure limiting rate coefficients, which are given in Table 2 and shown in Figure 3. Sehested et al.[9] carried out time-resolved experiments, where $\mathrm{CH}_{3} \mathrm{OCH}$ was monitored using UV absorption. However, the $\left[\mathrm{CH}_{3} \mathrm{OCH}_{2}\right]$ was so high that distinguishing $k_{1}$ from self-reaction, $k_{4}$, was not fully resolved and as such their $k_{1}$ values just reinforced those of Loucks and Laidler.[8] 
The $a b$ initio potential energy surface of R1 was calculated by Li et al.[10] and micro-canonical transition-state theory was then used to calculate $k_{1}^{\infty}(T)$, which is given in Table 2 and shown in Figure 3. Most recently, Gao et al.[11] carried out further calculations on R1, where the calculations were at a higher level and attention was given to the low frequency torsions / anharmonic effects and to tunnelling. Multi-structural canonical variational transition-state calculations were carried out and their $k_{1}^{\infty}(T)$ is given in Table 2 and shown in Figure 3.

Our present results with only the helium data - Table 1 - returned a barrier $\left(108.5 \mathrm{~kJ} \mathrm{~mol}^{-1}\right)$ with significant error $\left( \pm 8 \mathrm{~kJ} \mathrm{~mol}^{-1}\right.$ ) when analysed by MESMER. To improve the error in the barrier and the overall parameterisation, the data from Loucks and Laidler were also included in the MESMER calculation, where $k_{1}$ was obtained by digitizing their graphical data and converting it to absolute rate coefficients via a more recent determination of $k_{4},[31]$ the reference reaction in their experiments. The fit parameters from this MESMER analysis are given in Table 2, where it can be seen that the zero temperature barrier to dissociation, $\Delta E_{0,1}$, is defined with a small error $\left(112.5 \pm 0.6 \mathrm{~kJ} \mathrm{~mol}^{-1}\right)$. This value is in good agreement with our $a b$ initio calculation and the calculations by Gao et al.[11], see Table 2. Figure 4 shows a plot of the experimental rate coefficients vs the calculated rate coefficients. The plot shows that the scatter of the Loucks and Laidler data is small, in accordance with the low error in the barrier height when these data are included. The scatter for the Leeds data is greater, but the values are randomly distributed around the line with unit slope in Fig. 4; the direct measurements presented here confirm that no significant systematic error occurs in either the original data of Loucks and Laidler, or in its digitisation and conversion using the Maricq et al. [31] values for $k_{4}$. Figure 4 also shows the three direct measurements by Sehested et al.,[9] which are less compatible with the other data and have not been used in the fits. 


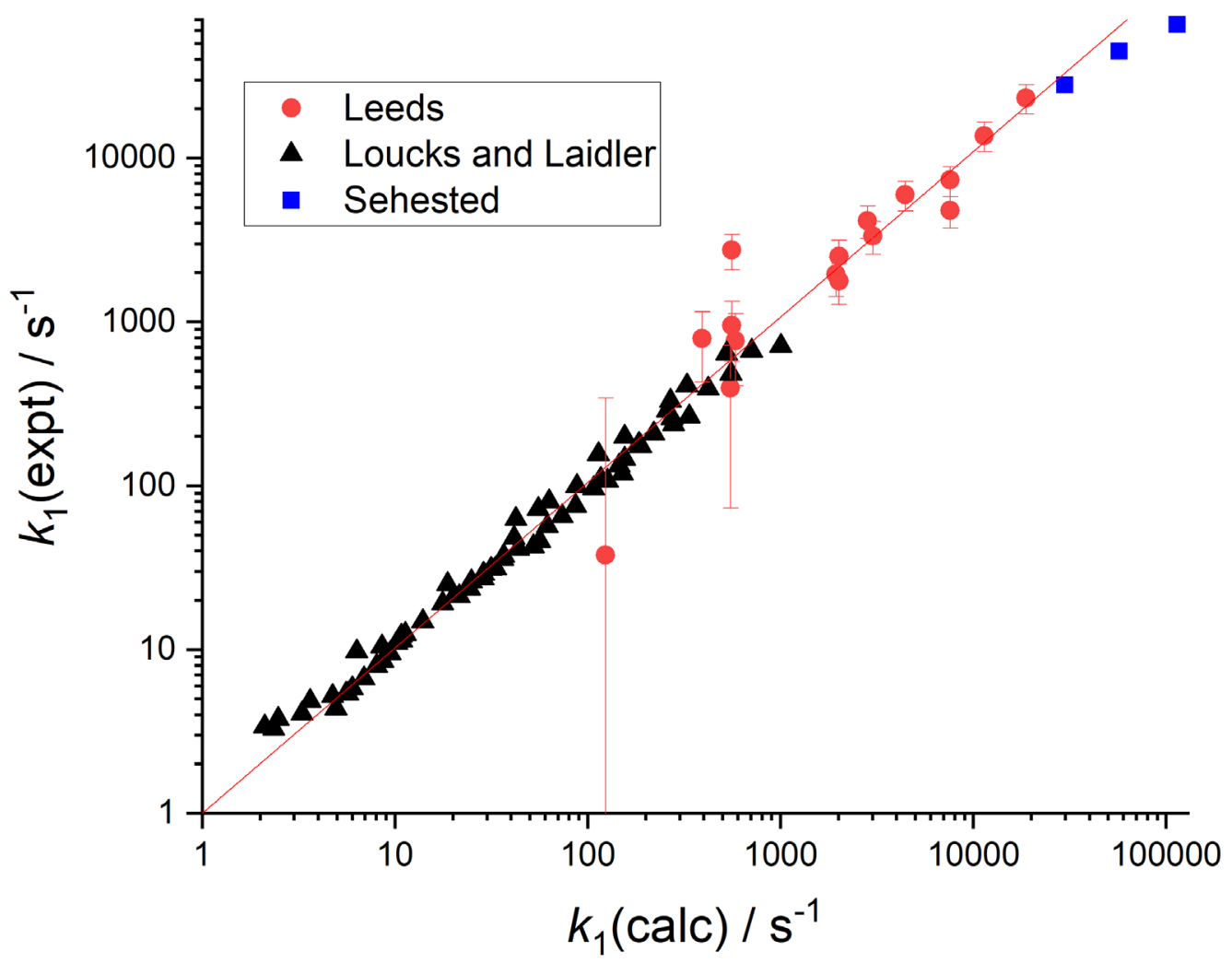

Figure 4. Plot of the experimental versus the MESMER best-fit calculated values for $k_{1}(T, p)$. The slope of the line through the data is equal to 1.01, which indicates good agreement. Also included in the plot are the Sehested et al. data.

In our MESMER analysis, energy transfer was adjusted with a temperature dependence, $<\Delta E_{\text {down, }}>\times(T / x)^{n}$, where $\mathrm{x}$ is either 298 for helium and nitrogen or 521 for dimethyl ether, the centre temperature in the Loucks and Laidler study. While the energy transfer parameters have significant errors (Table 2), there is a strong correlation between the values of $\left\langle\Delta E_{\text {down }}\right\rangle$ and $n$. When their covariance is propagated into the calculation of the overall uncertainty for a given bath gas at a specific temperature, the energy transfer is well-defined with an error of $\sim 10 \%$. The energy transfer values for helium are high but are in agreement with the value in our previous study of the reaction of $\mathrm{CH}_{3} \mathrm{OCH}_{2}$ with $\mathrm{O}_{2}$.[34]

In this MESMER analysis the two lowest frequencies are treated as hindered rotors for both $\mathrm{CH}_{3} \mathrm{OCH}_{2}$ and the transition-state, see Supplementary Information. When the MESMER analysis used a rigid rotor harmonic oscillator model for both $\mathrm{CH}_{3} \mathrm{OCH}_{2}$ and the transitionstate, the fit to the data was similar but $\Delta E_{0,1}$ increased by $2.5 \mathrm{~kJ} \mathrm{~mol}^{-1}$. This effect on $\Delta E_{0,1}$ of 
using hindered rotors in the fit is typical.[35] A fit to the high pressure limit from the MESMER analysis over the temperature range $300-1100 \mathrm{~K}$ gives $k_{1}^{\infty}(T)=2.9 \times 10^{12}(T / 300)^{2.5} \exp (-$ $\left.106.8 \mathrm{~kJ} \mathrm{~mol}^{-1} / \mathrm{R} T\right) \mathrm{s}^{-1}$.

From Table 2 and Figure 3, our values of $k_{1}^{\infty}$ compare most favourably with the result of Li et al.[10] Our MESMER result is heavily weighted by the Loucks and Laidler data, therefore the difference in Figure 3 between our $k_{1}^{\infty}$ and the original result by Loucks and Laidler is mainly from the more thorough master equation analysis compared with the limited LindemannHinshelwood extrapolation. The biggest difference in Figure 3 is with the results of Gao et al. who employed the most detailed theoretical analysis, using multi-structural variational transition-state theory; they predict a significantly faster $k_{1}^{\infty}$ at low temperatures. It is important to assess the origins of these differences in $k_{1}^{\infty}$. Our MESMER fitting is based on electronic structure calculations of the vibrational frequencies of the reactant and transition state and incorporates, as discussed above, two hindered internal rotors. Tunnelling was incorporated using an Eckart analysis, with an imaginary frequency of $767 \mathrm{~cm}^{-1}$. Since the reaction involves breaking a $\mathrm{C}-\mathrm{O}$ bond, the reduced mass is high and the effects of tunnelling small ( $25 \%$ at $500 \mathrm{~K})$. The barrier height was tuned in the fits to the experimental data, and the resulting value agrees well with our calculated barrier height (Table 2). As shown in Figure 4 , the best fit to the experimental data is good over a four order of magnitude change in $k_{1}$. As shown in Figure 3, the results of Gao et al. for $k_{1}^{\infty}$ substantially overestimate our best fit values; at $500 \mathrm{~K}$, the difference is over a factor of 10 . They used multistructural canonical variational transition state theory, incorporating anharmonicity; they also used two hindered internal rotors. They used multidimensional zero curvature- and small curvature- tunnelling approximations. Although they used a variational model, they noted that the variational effects were negligible. Despite the detailed nature of their model, their results agree poorly with experiment, as represented by our fitted high pressure rate constants. The activation energy in their modified Arrhenius expression is considerably lower than the barrier height, with the major contributions to this lowering deriving from the effects of tunnelling, which increase the rate constant at low $T$, and of multistructural effects, which reduce the rate constant at high $T$. It is difficult to criticise their analysis on theoretical grounds, but the poor agreement with an extensive experimental dataset necessitates rejection of their final results. 
In the intermediate temperature range, where autoignition occurs, the rate coefficients are comparable. At high temperatures, in the NTC range, all the studies have converged, see Figure 3, if the original Loucks and Laidler data are omitted and replaced with the results from the MESMER analysis.

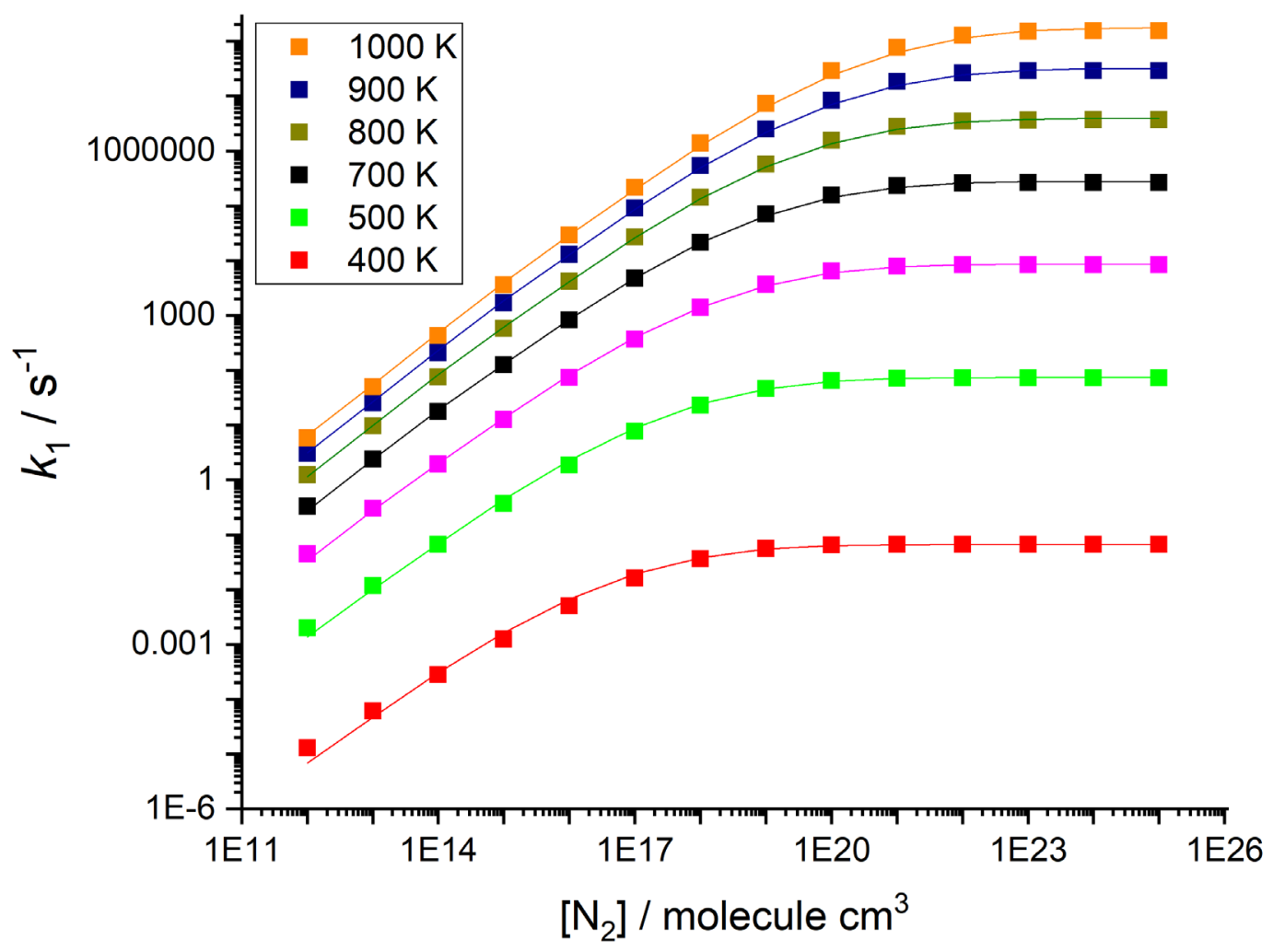

Figure 5. Simulated $k_{1}(T, p)$ for nitrogen, using the MESMER parameters in Table 2 . The line through the $k_{1}(T, p)$ data is from a Troe fit, see Table 2 for these parameters. 


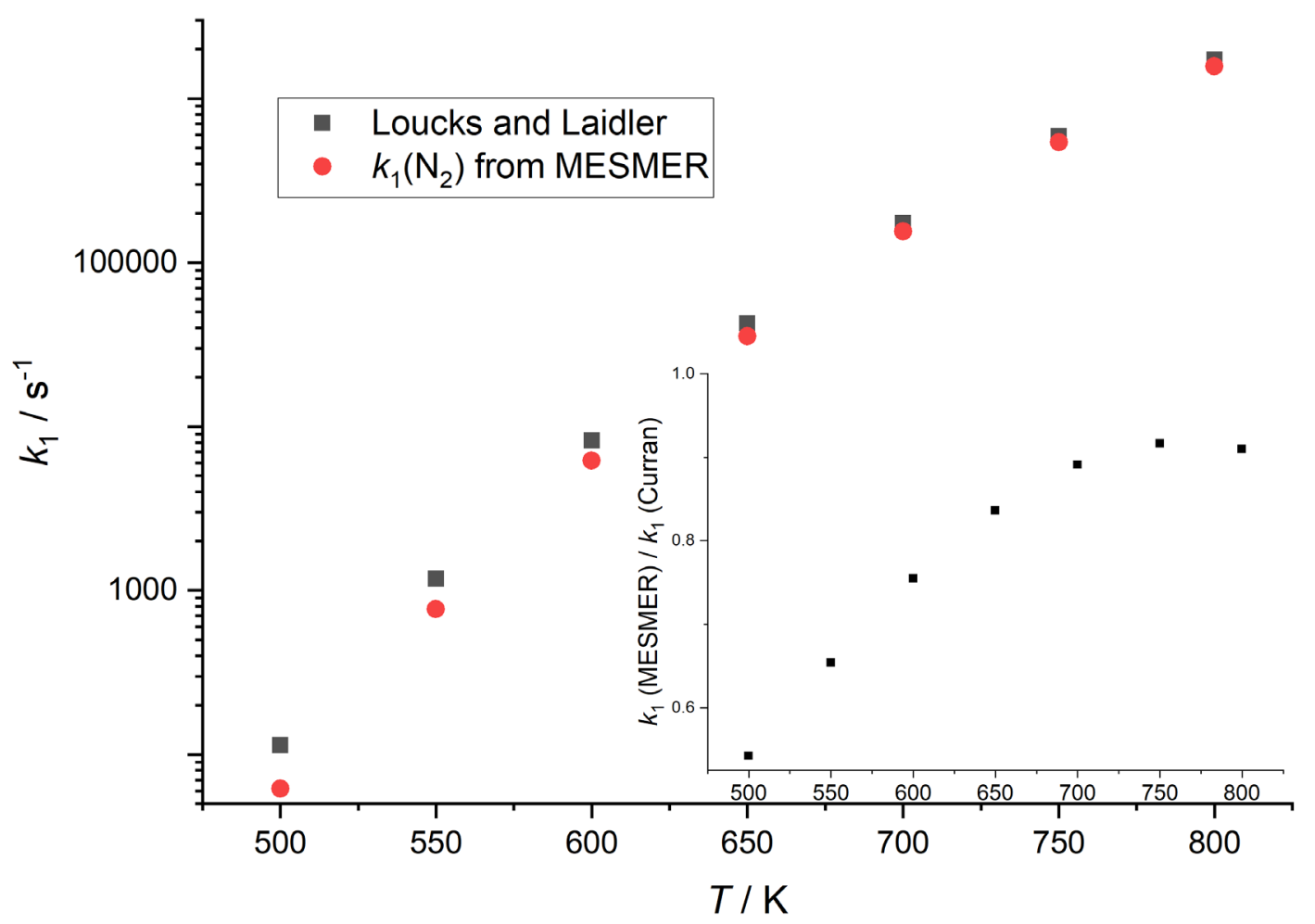

Figure 6. $k_{1}$ at $\left[\mathrm{N}_{2}\right]=10^{20}$ molecule $\mathrm{cm}^{-3}$ from MESMER versus Loucks and Laidler $k_{1}$ used in the dimethyl ether combustion model by Curran et al. [13] The inset shows $k_{1}$ (MESMER) / $k_{1}$ (Curran).

From Table 2, using the energy transfer values for nitrogen, MESMER simulation have been carried out to generate $k_{1}$ over a wide ranges of condition. These results have been parameterized using a Troe-type expression [36] and the parameters are given in Table 2. This fitting reproduces the MESMER result with an average error of 3\%, and is shown in Figure 5. There are large errors at the lowest pressures and temperatures, but these are under conditions that are not of practical importance. Over the temperature range $400-1000 \mathrm{~K}$ and for $\left[\mathrm{N}_{2}\right]=10^{17}-10^{21}$ molecule $\mathrm{cm}^{-3}$, the maximum difference from unity in the ratio of the Mesmer : Troe rate constants is $\sim 18 \%$. An alternative parameterization is via PLOG analysis and this is presented in the $\mathrm{SI}$ for all bath gases considered in the present study, $\mathrm{He}$, $\mathrm{CH}_{3} \mathrm{OCH}_{3}$ and $\mathrm{N}_{2}$.

The dimethyl ether combustion model developed by Curran et al. [15] was based on experiments carried out in a flow reactor using nitrogen as the buffer gas over the temperature and pressure ranges: $550-850 \mathrm{~K}$ and $12-18 \mathrm{~atm}$. Assuming $\left[\mathrm{N}_{2}\right]$ equal to $10^{20}$ 
molecule $\mathrm{cm}^{-3}$ for the experiments of Curran et al.,[15] the values for $k_{1}$ using our MESMER model are compared in Figure 6 with the values from Curran et al., which assumed the original Loucks and Laidler high pressure limiting Arrhenius expression. As can be seen in Figure 6, the values of $k_{1}$ are within a factor of three over the whole temperature range, and in good agreement at $\sim 800 \mathrm{~K}$. Therefore the dimethyl ether model by Fischer et al. [13] is little affected by our values for $k_{1}$ at the reactor pressures used by Curran et al.,[15] but this will not necessarily be true for lower pressures.

While the current experiments on their own do not determine the heat of formation of $\mathrm{CH}_{3} \mathrm{OCH}_{2}$, it can be assigned by considering the study of Gao et al.[11] From Table 2, the two calculations for $\Delta E_{0,1}$ from $G$ ao et al. are higher and lower than our value, but the associated reaction energy at $0 \mathrm{~K}$ from these calculations are $28.5(\mathrm{G} 4)$ and $27.1 \mathrm{~kJ} \mathrm{~mol}^{-1}(\mathrm{QCISD}(\mathrm{T}) /$ augcc-pVTZ//MP2/TZVP); so the variation in reaction energy with level of calculation is significantly smaller than the variation in the barrier energies. If these two values for the heat of reaction are combined with the well-known zero $\mathrm{K}$ heats of formation of $\mathrm{CH}_{3}(149.9 \mathrm{~kJ}$ $\mathrm{mol}^{-1}$ ) and $\mathrm{CH}_{2} \mathrm{O}\left(-105.38 \mathrm{~kJ} \mathrm{~mol}^{-1}\right)$ (uncertainties less than $\left.0.1 \mathrm{~kJ} \mathrm{~mol}^{-1}[37,38]\right)$, then the heat of formation of $\mathrm{CH}_{3} \mathrm{OCH}_{2}\left(\Delta_{\mathrm{f}} H^{0}(\mathrm{O} \mathrm{K})\right)$ is equal to 16.0 or $17.3 \mathrm{~kJ} \mathrm{~mol}^{-1}$. The uncertainty in this value is down to the reliability of the $a b$ initio calculation, which we estimate to be $\sim 4 \mathrm{~kJ} \mathrm{~mol}^{-}$ $1,1 \sigma$. The only previous calculation on the heat of formation $\mathrm{CH}_{3} \mathrm{OCH}_{2}$ is by $\mathrm{Good}$ and Francisco,[39] who calculated $\Delta_{\mathrm{f}} H^{0}(0 \mathrm{~K})=17.6 \mathrm{~kJ} \mathrm{~mol}^{-1}$ using a lower level of theory. The enthalpy of formation of methoxymethyl at $298 \mathrm{~K}$ was discussed by Gao et al., but using outdated enthalpies of formation for methyl and formaldehyde.

\section{Conclusions}

Time-resolved experiments have been carried out to investigate the rate coefficients for unimolecular decomposition of $\mathrm{CH}_{3} \mathrm{OCH}_{2}, k_{1}$; the $\mathrm{HCHO}$ product of this reaction was monitored using laser induced fluorescence. The kinetics were determined over a range of temperatures and pressures. These results were analysed using a master equation approach (MESMER) and, to better define the system, the data from Loucks and Laidler [8]were also included in this analysis, after appropriate modification. The reaction barrier was identified with high precision $\left(\Delta E_{0,1}=(112.5 \pm 0.6) \mathrm{kJ} \mathrm{mol}^{-1}\right)$, which is in good agreement with our $a b$ 
initio calculations. MESMER simulations with $\mathrm{N}_{2}$ as the bath gas, using energy transfer parameters from a previous study, allowed representations of $k_{1}(T, p)$ to be determined using both a Troe expression and the PLOG approach, allowing the results to be used in combustion modelling.

\section{Acknowledgement}

A.J.E. acknowledges support from the Academy of Finland, Grant Nos. 130347, 125540, and 288377. 


\section{REFERENCES}

[1] M.J. Pilling, S.H. Robertson, P.W. Seakins, J. Chem. Soc., Faraday Trans. 91 (1995) 4179.

[2] J. Zador, C.A. Taatjes, R.X. Fernandes, Prog. Energy Combust. Sci. 37 (2011) 371.

[3] S.H. Park, C.S. Lee, Prog. Energy Combust. Sci. 39 (2013) 147.

[4] G. Thomas, B. Feng, A. Veeraragavan, M.J. Cleary, N. Drinnan, Fuel Process. Technol. 119 (2014) 286.

[5] C. Bansch, J. Kiecherer, M. Szori, M. Olzmann, J. Phys. Chem. A 117 (2013) 8343.

[6] S.A. Carr, T.J. Still, M.A. Blitz, A.J. Eskola, M.J. Pilling, P.W. Seakins, et al, J. Phys. Chem. A 117 (2013) 11142.

[7] M. Kawade, A. Sharma, D. Srinivas, A. Saha, H.P. Upadhyaya, A. Kumar, et al, Chem. Phys. Lett. 706 (2018) 558.

[8] L.F. Loucks, K.J. Laidler, Can. J. Chem. 45 (1967) 2767.

[9] J. Sehested, K. Sehested, J. Platz, H. Egsgaard, O.J. Nielsen, Int. J. Chem. Kinet. 29 (1997) 627.

[10] Q.S. Li, Y. Zhang, S. Zhang, J. Phys. Chem. A 108 (2004) 2014.

[11] J. Gao, Y. Guan, J. Lou, H. Ma, J. Song, Combust. Flame 197 (2018) 243.

[12] H.J. Curran, W.J. Pitz, C.K. Westbrook, P. Dagaut, J.C. Boettner, M. Cathonnet, Int. J. Chem. Kinet. 30 (1998) 229.

[13] S.L. Fischer, F.L. Dryer, H.J. Curran, Int. J. Chem. Kinet. 32 (2000) 713.

[14] P. Dagaut, J. Luche, M. Cathonnet, Combust. Sci. Technol. 165 (2001) 61.

[15] H.J. Curran, S.L. Fischer, F.L. Dryer, Int. J. Chem. Kinet. 32 (2000) 741.

[16] D.G. Potter, M.A. Blitz, P.W. Seakins, Chem. Phys. Lett. 730 (2019) 213.

[17] A.V. Baklanov, L.N. Krasnoperov, J. Phys. Chem. A 105 (2001) 97.

[18] T.J. Gravestock, M.A. Blitz, W.J. Bloss, D.E. Heard, ChemPhysChem 11 (2010) 3928.

[19] C. Brackmann, J. Nygren, X. Bai, Z.S. Li, H. Bladh, B. Axelsson, et al, Spectroc. Acta Pt. AMolec. Biomolec. Spectr. 59 (2003) 3347.

[20] D.R. Glowacki, C.H. Liang, C. Morley, M.J. Pilling, S.H. Robertson, J. Phys. Chem. A 116 (2012) 9545.

[21] M.J. Frisch, G.W. Trucks, H.B. Schlegel, G.E. Scuseria, M.A. Robb, J.R. Cheeseman, et al, Gaussian 09. Gaussian, Inc., Wallingford CT, 2016, 2009.

[22] H.J. Werner, P.J. Knowles, G. Knizia, F.R. Manby, M. Schutz, Wiley Interdiscip. Rev.-Comput. Mol. Sci. 2 (2012) 242.

[23] S.H. Robertson, D.R. Glowacki, C.-H. Liang, C. Morley, R.J. Shannon, M.A. Blitz, et al. MESMER (Master Equation Solver for Multi-Energy Well Reactions), 2008-2013; an Object Oriented C++ Program Implementing Master Equation Methods for Gas Phase Reactions with Arbitrary Multiple Wells. [cited 2019] Available from:

http://sourceforge.net/projects/mesmer.

[24] S. Sharma, S. Raman, W.H. Green, J. Phys. Chem. A 114 (2010) 5689.

[25] S.H. Robertson, Part 2: Collisional Energy Transfer and The Master Equation, ELSEVIER, 2019.

[26] K.G. Joback, R.C. Reid, Chem. Eng. Commun. 57 (1987) 233.

[27] C.W. Gao, J.W. Allen, W.H. Green, R.H. West, Comput. Phys. Commun. 203 (2016) 212.

[28] J.V. Michael, D.F. Nava, W.A. Payne, L.J. Stief, J. Chem. Phys. 70 (1979) 3652.

[29] A.J. Eskola, S.A. Carr, M.A. Blitz, M.J. Pilling, P.W. Seakins, Chem. Phys. Lett. 487 (2010) 45.

[30] B. Ghosh, D.K. Papanastasiou, J.B. Burkholder, J. Chem. Phys. 137 (2012) 12.

[31] M.M. Maricq, J.J. Szente, J.D. Hybl, J. Phys. Chem. A 101 (1997) 5155.

[32] A. Shepp, J. Chem. Phys. 24 (1956) 939.

[33] T.H. Speak, M.A. Blitz, D. Stone, P.W. Seakins, Atmos. Meas. Tech. 13 (2020) 839.

[34] A.J. Eskola, S.A. Carr, R.J. Shannon, B. Wang, M.A. Blitz, M.J. Pilling, et al, J. Phys. Chem. A 118 (2014) 6773. 
[35] D.J. Medeiros, M.A. Blitz, L. James, T.H. Speak, P.W. Seakins, J. Phys. Chem. A 122 (2018) 7239.

[36] J. Troe, V.G. Ushakov, Z. Phys. Chem. (Muenchen, Ger.) 228 (2014) 1.

[37] B. Ruscic, Int. J. Quantum Chem. 114 (2014) 1097.

[38] B. Ruscic, D.H. Bross. Active Thermochemical Tables (ATCT) values based on ver. 1.122g of the Thermochemical Network [cited 2019] Available from: https://atct.anl.gov/

[39] D.A. Good, J.S. Francisco, Chem. Phys. Lett. 266 (1997) 512. 


\section{Supplementary Information}

The MESMER fit to the data is given below, where the first 16 points are Leeds and the rest are Loucks and Laidler. This is followed by the best fit parameters together with the correlation matrix. PLOG parameterization of $k_{1}(T, p)$ is also for the buffers gases: $\mathrm{CH}_{3} \mathrm{OCH}_{3}$, He and $\mathrm{N}_{2}$.

The $x m l$ MESMER input file for $\mathrm{CH}_{3} \mathrm{OCH}_{2}$ is given at the end: $\mathrm{CH}_{3} \mathrm{OCH}_{2}$. $\mathbf{x m l}$

\section{MESMER}

$\begin{array}{lll} & \text { Exp. } & \text { Cal. } \\ \text { Temperature Concentration } & \text { Coeff } & \text { Coeff. }\end{array}$

$\begin{array}{llrr}673 & 1.05 \mathrm{E}+17 & 1772 & 2011.63 \\ 673 & 1.05 \mathrm{E}+17 & 2520 & 2011.63 \\ 673 & 7.87 \mathrm{E}+17 & 7349 & 7590.4 \\ 673 & 7.87 \mathrm{E}+17 & 4777 & 7590.4 \\ 673 & 1.58 \mathrm{E}+18 & 13700 & 11400.9 \\ 673 & 4.01 \mathrm{E}+18 & 23275 & 18785.5 \\ 622 & 1.14 \mathrm{E}+17 & 951 & 559.68 \\ 622 & 1.14 \mathrm{E}+17 & 2747 & 559.68 \\ 622 & 8.50 \mathrm{E}+17 & 1948 & 1943.29 \\ 622 & 1.93 \mathrm{E}+18 & 3347 & 3015.4 \\ 622 & 1.70 \mathrm{E}+18 & 4151 & 2824.38 \\ 622 & 4.34 \mathrm{E}+18 & 5969 & 4438.47 \\ 573 & 1.23 \mathrm{E}+17 & 37.6921 & 123.697 \\ 573 & 9.24 \mathrm{E}+17 & 791 & 392.47 \\ 573 & 2.10 \mathrm{E}+18 & 767 & 584.095 \\ 573 & 1.85 \mathrm{E}+18 & 396 & 550.75 \\ 573 & 5.53 \mathrm{E}+16 & 155.681 & 113.469 \\ 573 & 9.13 \mathrm{E}+16 & 199.635 & 155.502 \\ 573 & 1.21 \mathrm{E}+17 & 179.571 & 184.114 \\ 573 & 2.49 \mathrm{E}+17 & 238.361 & 279.347 \\ 573 & 3.48 \mathrm{E}+17 & 264.994 & 335.367 \\ 573 & 5.42 \mathrm{E}+17 & 391.053 & 422.194 \\ 573 & 8.48 \mathrm{E}+17 & 641.558 & 524.977 \\ 573 & 9.47 \mathrm{E}+17 & 483.321 & 552.864 \\ 573 & 1.65 \mathrm{E}+18 & 665.635 & 708.163 \\ 573 & 4.03 \mathrm{E}+18 & 714.884 & 1002.96 \\ 543 & 6.16 \mathrm{E}+16 & 62.5794 & 42.469 \\ 543 & 9.63 \mathrm{E}+16 & 72.1824 & 55.3289 \\ 543 & 1.20 \mathrm{E}+17 & 80.2475 & 62.8739\end{array}$




$\begin{array}{rrrr}543 & 2.22 \mathrm{E}+17 & 99.1817 & 87.8597 \\ 543 & 3.88 \mathrm{E}+17 & 110.263 & 116.609 \\ 543 & 6.77 \mathrm{E}+17 & 118.422 & 151.486 \\ 543 & 7.16 \mathrm{E}+17 & 146.363 & 155.281 \\ 543 & 1.12 \mathrm{E}+18 & 174.756 & 187.947 \\ 543 & 1.65 \mathrm{E}+18 & 208.656 & 219.693 \\ 543 & 2.57 \mathrm{E}+18 & 287.363 & 258.631 \\ 543 & 2.88 \mathrm{E}+18 & 330.698 & 268.925 \\ 543 & 3.05 \mathrm{E}+18 & 257.888 & 274.019 \\ 543 & 5.32 \mathrm{E}+18 & 408.725 & 326.788 \\ 521 & 6.80 \mathrm{E}+16 & 25.1082 & 18.793 \\ 521 & 1.12 \mathrm{E}+17 & 25.9905 & 24.8604 \\ 521 & 1.48 \mathrm{E}+17 & 28.9611 & 28.8608 \\ 521 & 1.75 \mathrm{E}+17 & 31.0324 & 31.5023 \\ 521 & 2.32 \mathrm{E}+17 & 35.7944 & 36.2729 \\ 521 & 3.42 \mathrm{E}+17 & 41.1922 & 43.7785 \\ 521 & 5.04 \mathrm{E}+17 & 42.7381 & 52.2771 \\ 521 & 5.97 \mathrm{E}+17 & 45.9003 & 56.251 \\ 521 & 7.46 \mathrm{E}+17 & 56.7303 & 61.8047 \\ 521 & 1.16 \mathrm{E}+18 & 65.2853 & 73.7225 \\ 521 & 1.82 \mathrm{E}+18 & 75.3035 & 86.7095 \\ 521 & 3.55 \mathrm{E}+18 & 96.564 & 107.42 \\ 521 & 6.54 \mathrm{E}+18 & 107.353 & 126.898 \\ 521 & 1.21 \mathrm{E}+19 & 132.683 & 145.853 \\ 498 & 6.02 \mathrm{E}+16 & 9.74255 & 6.3363 \\ 498 & 1.05 \mathrm{E}+17 & 10.4393 & 8.55305 \\ 498 & 1.83 \mathrm{E}+17 & 12.4644 & 11.3135 \\ 498 & 2.86 \mathrm{E}+17 & 14.8824 & 13.9261 \\ 498 & 4.99 \mathrm{E}+17 & 19.0841 & 17.7129 \\ 498 & 6.97 \mathrm{E}+17 & 21.2164 & 20.236 \\ 498 & 8.25 \mathrm{E}+17 & 21.2164 & 21.571 \\ 498 & 1.15 \mathrm{E}+18 & 23.587 & 24.3351 \\ 498 & 1.90 \mathrm{E}+18 & 27.2065 & 28.7098 \\ 498 & 3.14 \mathrm{E}+18 & 31.3092 & 33.2277 \\ 498 & 4.64 \mathrm{E}+18 & 37.3828 & 36.7308 \\ 498 & 8.10 \mathrm{E}+18 & 47.9372 & 41.5857 \\ 473 & 7.92 \mathrm{E}+16 & 3.39853 & 2.10681 \\ 473 & 9.88 \mathrm{E}+16 & 3.28315 & 2.35286 \\ 473 & 1.11 \mathrm{E}+17 & 3.77825 & 2.48616 \\ 473 & 2.04 \mathrm{E}+17 & 4.0578 & 3.30191 \\ 473 & 2.55 \mathrm{E}+17 & 4.84496 & 3.63763 \\ 473 & 5.55 \mathrm{E}+17 & 4.35803 & 4.95328 \\ 473 & 4.97 \mathrm{E}+17 & 5.20344 & 4.75389 \\ 473 & 7.77 \mathrm{E}+17 & 5.3863 & 5.58086 \\ 473 & 8.22 \mathrm{E}+17 & 5.3863 & 5.68671 \\ 473 & 9.70 \mathrm{E}+17 & 5.78483 & 6.00809 \\ 473 & 1.52 \mathrm{E}+18 & 6.65718 & 6.89523 \\ 473 & 2.65 \mathrm{E}+18 & 7.9486 & 8.01644 \\ 473 & 5.40 \mathrm{E}+18 & 8.53671 & 8.67736 \\ 473 & 1.19 \mathrm{E}+18 & 9.49053 & 9.42061 \\ 473 & 1.26 \mathrm{E}+19 & 10.9469 & 10.2193 \\ & 11.3316 & 10.8536\end{array}$


$\begin{array}{lll}\text { Chi^}^{\wedge} 2 & = & 72.4903\end{array}$

Best fit parameters:

$\begin{array}{llrlr}\mathrm{CH} 3 \mathrm{OCH} 2: \text { deltaEDown:He } & & 195.801 & +/- & 320.849 \\ \mathrm{CH} 3 \mathrm{OCH} 2: \text { deltaEDown:CH3OCH3 } & = & 871.511 & +/- & 611.272 \\ \mathrm{CH} 3 O \mathrm{CH} 2: \text { deltaEDownTExponent:He } & = & 0.769149 & +/- & 2.0268 \\ \mathrm{CH} 3 O C H 2: \text { deltaEDownTExponent:CH3OCH3 } & = & -0.87181 & +/- & 1.00524 \\ \text { TS1:ZPE } & = & 128.512 & +/- & 0.581291\end{array}$

\section{CORRELATION MATRIX}

\begin{tabular}{|c|c|c|c|c|}
\hline Correlation & & efficients: & & \\
\hline $\mathrm{CH} 3 \mathrm{OCH}$ :deltaEDown: $\mathrm{CH} 3 \mathrm{OCH} 3$ & & CH3OCH2:deltaEDown:He & $=$ & 0.134329 \\
\hline CH3OCH2:deltaEDownTExponent:He & & $\mathrm{CH} 3 \mathrm{OCH} 2$ :deltaEDown:He & $=$ & -0.99796 \\
\hline $\mathrm{CH} 3 \mathrm{OCH}$ 2:deltaEDownTExponent:He & & $\mathrm{CH} 3 \mathrm{OCH}$ 2:deltaEDown: $\mathrm{CH} 3 \mathrm{OCH} 3$ & $=$ & -0.10237 \\
\hline $\mathrm{CH} 3 \mathrm{OCH} 2$ :deltaEDownTExponent:CH3OCH3 & & $\mathrm{CH} 3 \mathrm{OCH}$ 2:deltaEDown:He & $=$ & -0.11253 \\
\hline $\mathrm{CH} 3 \mathrm{OCH} 2$ :deltaEDownTExponent: $\mathrm{CH} 3 \mathrm{OCH} 3$ & & $\mathrm{CH} 3 \mathrm{OCH} 2$ :deltaEDown: $\mathrm{CH} 3 \mathrm{OCH} 3$ & $=$ & -0.98254 \\
\hline $\mathrm{CH} 3 \mathrm{OCH} 2$ :deltaEDownTExponent: $\mathrm{CH} 3 \mathrm{OCH} 3$ & & CH3OCH2:deltaEDownTExponent:He & $=$ & 0.085754 \\
\hline TS1:ZPE & & $\mathrm{CH} 3 \mathrm{OCH}$ : deltaEDown: $\mathrm{He}$ & $=$ & 0.177591 \\
\hline TS1:ZPE & & $\mathrm{CH} 3 \mathrm{OCH} 2$ :deltaEDown: $\mathrm{CH} 3 \mathrm{OCH} 3$ & $=$ & 0.756393 \\
\hline TS1:ZPE & & $\mathrm{CH} 3 \mathrm{OCH}$ 2:deltaEDownTExponent:He & $=$ & -0.13534 \\
\hline TS1:ZPE & & $\mathrm{CH} 3 \mathrm{OCH}$ 2: deltaEDownTExponent:CH3 & & -0.63363 \\
\hline
\end{tabular}




\section{PLOG}

\begin{tabular}{|c|c|c|c|c|c|c|}
\hline Bath & gas & is & $\mathrm{CH} 3 \mathrm{OCH}$ & & & \\
\hline units(length & $=$ & 'cm', & quantity & $=$ & 'mole') & \\
\hline \multirow[t]{8}{*}{ pdep_arrhenius(' $\mathrm{CH} 3 \mathrm{OCH} 2$} & $=>$ & $\mathrm{CH} 2 \mathrm{O}$ & + & $\mathrm{CH}^{\prime}$, & & \\
\hline & {$[($} & 0.001, & 'atm'), & $5.66189 e+52$ & -13.7666 & 34561.6], \\
\hline & {$[($} & 0.01, & 'atm'), & $3.22796 e+52$ & -13.3182, & 35451.7], \\
\hline & {$[($} & 0.1 & 'atm'), & $2.67855 e+49$ & -11.9866 & 35643.6], \\
\hline & {$[($} & 1 & 'atm'), & 1.18294e+42, & -9.34681 & 34514.1], \\
\hline & {$[($} & 10 & 'atm'), & 1.06617e+31, & -5.61002 & 32056.1], \\
\hline & {$[($} & 100 & 'atm'), & $2.60276 e+19$ & -1.77664 & 29124.8], \\
\hline & {$[($} & 1000, & 'atm'), & $1.00434 e+11$ & 0.967752 & 26874]) \\
\hline Bath & gas & is & $\mathrm{He}$ & & & \\
\hline units(length & $=$ & 'cm', & quantity & $=$ & 'mole') & \\
\hline \multirow[t]{8}{*}{ pdep_arrhenius(' $\mathrm{CH} 3 \mathrm{OCH} 2$} & $=>$ & $\mathrm{CH} 2 \mathrm{O}$ & + & $\mathrm{CH} 3$, & & \\
\hline & {$[($} & 0.001 & 'atm'), & $3.56127 e+36$ & -8.51999 & 30552], \\
\hline & {$[($} & 0.01, & 'atm'), & 3.47613e+36 & -8.17061 & 31257.8], \\
\hline & {$[($} & 0.1 & 'atm'), & 7.28559e+34, & -7.30714 & 31624.4], \\
\hline & {$[($} & 1, & 'atm'), & $2.50608 \mathrm{e}+30$ & -5.59772 & 31216.2], \\
\hline & {$[($} & 10 & 'atm'), & $6.97338 e+22$ & -2.97588 & 29740.6], \\
\hline & {$[($} & 100 & 'atm'), & $2.75721 e+14$ & -0.172067 & 27724.1], \\
\hline & {$[($} & 1000 & 'atm'), & $5.34256 e+08$ & 1.70146, & 26233.6]) \\
\hline Bath & gas & is & N2 & & & \\
\hline units(length & $=$ & 'cm', & quantity & $=$ & 'mole') & \\
\hline \multirow[t]{8}{*}{ pdep_arrhenius(' $\mathrm{CH} 3 \mathrm{OCH} 2$} & $=>$ & $\mathrm{CH} 2 \mathrm{O}$ & + & $\mathrm{CH} 3 '$ & & \\
\hline & {$[($} & 0.001, & 'atm'), & 5.13565e+39, & -9.48044 & 31404.5], \\
\hline & {$[($} & 0.01, & 'atm'), & 3.54673e+39, & -9.07744 & 32166.3], \\
\hline & {$[($} & 0.1 & 'atm'), & $1.70294 \mathrm{e}+37$ & -8.0086 & 32436.6], \\
\hline & {$[($} & 1, & 'atm'), & $3.20089 e+31$ & -5.90578 & 31681.7], \\
\hline & {$[($} & 10, & 'atm'), & 5.11864e+22, & -2.90525 & 29800.9], \\
\hline & {$[($} & 100 & 'atm'), & 7.46442e+13, & 0.021111 & 27602.8], \\
\hline & {$[($} & 1000 & 'atm'), & $2.51763 e+08$ & 1.8091, & 26150.2]) \\
\hline
\end{tabular}

\section{$\mathrm{CH}_{3} \mathrm{OCH}_{2}$ xml}

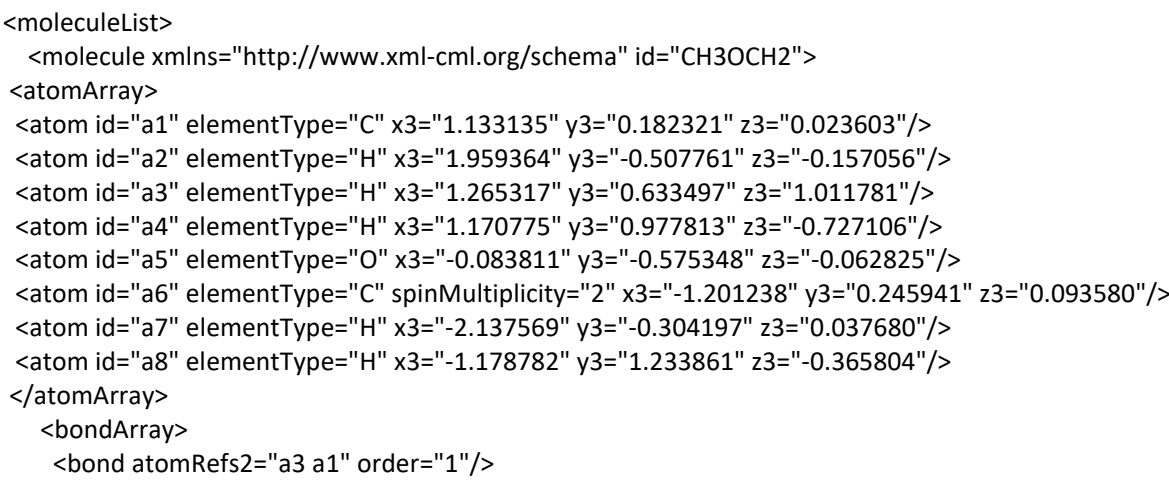


<bond atomRefs2="a7 a6" order="1"/> <bond atomRefs2="a2 a1" order="1"/>

<bond id="b1" atomRefs2="a5 a1" order="1"/>

<bond id="b2" atomRefs2="a5 a6" order="1"/>

<bond atomRefs $2=" a 8$ a6" order="1"/>

<bond atomRefs2="a1 a4" order="1"/>

$<$ /bondArray>

$<$ propertyList>

$<$ property title="program">

<scalar>Gaussian 09, Revision D.01</scalar>

$</$ property $>$

$<$ property title="basis" $>$

<scalar >6-31+G(d,p) $(6 \mathrm{D}, 7 \mathrm{~F})</$ scalar $>$

$<$ property $>$

$<$ property title="method" $>$

$<$ scalar $>$ m062x $<$ /scalar $>$

$<$ property $>$

$<$ property title="File Format" $>$

$<$ scalar>g03</scalar $>$

$</$ property $>$

$<$ property title="Energy" dictRef="me:ZPE">

<scalar units="kJ/mol" >15.97</scalar >

$</$ property $>$

$<$--- <property dictRef="me:vibFreqs"> -->

<!-- <array units="cm-1">199.43 281.27 502.82892 .661135 .451259 .581310 .361428 .281456 .371724 .451769 .00

$1803.411816 .703523 .423578 .573691 .433721 .753760 .59</$ array $>-->$

$<$--- </property>-->

<property title="Rotational Constants" dictRef="me:rotConsts">

<array units="cm-1">1.468 $0.3540 .305<$ /array $>$

$</$ property $>$

$<$ scalar $>0.86</$ scalar $>$

<property dictRef="me:frequenciesScaleFactor">

$</$ property $>$

<property title="Hessian" dictRef="me:hessian">

<matrix rows="24" matrixType="squareSymmetricLT" units="Hartree/Bohr2">0.79827926 -0.05787253 0.84101881 -0.00540215 $0.006586240 .81400113-0.278878370 .182479610 .046553980 .302658820 .18765979-0.24288937-0.04117453-0.19090282$

$0.240082410 .04743388-0.04154478-0.09366654-0.049738230 .044195870 .07979925-0.07940451-0.00834751-0.03544763$ $0.008459690 .010391890 .031832510 .08105183-0.01037969-0.13863855-0.13368942-0.00620638-0.00594536-0.02625512$ $0.022937230 .13740048-0.03300529-0.13055498-0.38509290-0.003816780 .003918030 .000334200 .040553290 .14587646$ $0.39499166-0.076537800 .000597470 .006888430 .005827820 .02088519-0.025694260 .002726170 .00417794-0.00096626$ $0.07711457-0.00071096-0.267005140 .18255902-0.00495411-0.012440620 .020612020 .001370590 .01794079-0.01766457$ $0.013335750 .278992570 .004445240 .17983164-0.258535360 .00143781-0.011585910 .00975547-0.000259440 .03277297$ $-0.01853782-0.00663748-0.196644020 .25730761-0.30235601-0.16711055-0.02136813-0.03196787-0.02225366-0.00267713$ $-0.01887827-0.01351367-0.00382374-0.01586321-0.012827330 .001107810 .72242512-0.09020690-0.20058248-0.01689578$ $0.023318600 .023588760 .00366800-0.02372859-0.01072119-0.00272916-0.03407156-0.01917162-0.00317189-0.00971125$ $0.44542320-0.01316817-0.01494517-0.073707770 .005393780 .005000720 .00267136-0.03446643-0.019151890 .00721874$ $0.025498050 .012347830 .00969105-0.016729420 .039321610 .07252495-0.063200160 .040752210 .00951861-0.00513742$ $-0.00455312-0.001026830 .006575180 .003248450 .000731030 .006179510 .003840060 .00000621-0.328321400 .12237525$ $0.053395260 .82788951-0.029484190 .010285820 .00431495-0.00465003-0.00401871-0.00069831-0.00318124-0.00003478$ $0.00140831-0.005299370 .00126574-0.001127800 .19859715-0.24422995-0.035983730 .027102080 .80163750-0.00177177$ $0.00208810-0.005088360 .00010414-0.000229270 .00090913-0.002434910 .000448760 .001273000 .00172414-0.00107107$ $0.000385060 .04222984-0.01901261-0.02816136-0.04317663-0.134346430 .15923118-0.003383340 .004484800 .00098715$ $-0.00101204-0.00098619-0.000157110 .000125790 .000172490 .000225920 .000210020 .00023424-0.00012222-0.02187857$ $-0.02044623-0.00566446-0.36090329-0.16434143-0.008107900 .379277690 .00367206-0.00472804-0.000828780 .00125023$ $0.001530090 .000133200 .000299570 .00004378-0.000139790 .000346290 .00010527-0.000014970 .026085100 .02177575$ $0.00175179-0.17966863-0.186337770 .005510890 .174684310 .171908160 .00241240-0.000796600 .001948130 .00015479$ $0.000117000 .000196900 .00005658-0.00006820-0.00004636-0.00032897-0.000346170 .000038160 .002212320 .00731404$ $0.00167811-0.03817613-0.01795105-0.025983300 .017654480 .002184320 .016735400 .005480930 .00501650-0.00173026$ $0.00004938-0.000241070 .00002717-0.00065587-0.000436370 .000101820 .00034293-0.000288240 .00002208-0.00315979$ $0.03247069-0.01425861-0.08308193-0.018742970 .011433090 .00756376-0.026668930 .016014520 .07346059-0.00267757$ $0.00253895-0.00087171-0.000335100 .00009281-0.000110880 .00025807-0.00004517-0.000114300 .000028290 .00031302$ $-0.000060020 .00073422-0.016082470 .01165884-0.01309631-0.378567850 .146611630 .00619801-0.004297240 .00954666$ $0.008890400 .39604796-0.00094415-0.000664450 .00014167-0.00008948-0.000241920 .000000230 .000166020 .00006644$ $-0.00014053-0.000483660 .00020697-0.00010417-0.00095155-0.008494200 .008084910 .018728470 .18438406-0.10256536$ $-0.00481585-0.008596670 .00543296-0.01160981-0.166660230 .08915028</$ matrix $>$

$</$ property $>$

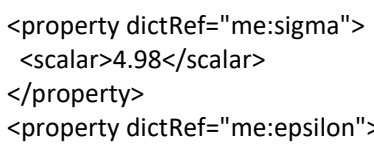


$<$ scalar $>329.669</$ scalar $>$
$<$ property $>$

<property title="Symmetry Number" dictRef="me:symmetryNumber">

$<$ scalar $>1<$ scalar $>$

$<$ property $>$

$<$ property dictRef="me:spinMultiplicity">

$<$ scalar $>2<$ scalar $>$

$<$ property dictRef="me:MW">

$<$ scalar units="amu" $>45<$ scalar $>$

$</$ property $>$

$</$ property $>$

$<$ propertyList $>$

$<$ me:DOSCMethod $>$ Classical rotors</me:DOSCMethod $>$

$<$ me:ExtraDOSCMethod xsi:type="HinderedRotorQM1D">

$<$ me:bondRef $>$ b1</me:bondRef $>$

<me:HinderedRotorPotential format="numerical" units="kJ/mol" expansionSize="7" useSineTerms="yes" scale="1">

$<$ me:PotentialPoint angle=" $0 \quad$ "potential= " 0

$<$ me:PotentialPoint angle=" $60 \quad$ "potential= " 6.415658673

$<$ me:PotentialPoint angle=" $90 \quad$ "potential= " 2.985975899

$<$ me:PotentialPoint angle=" $120 \quad$ "potential= " $\quad-0.018026683$

$<$ me:PotentialPoint angle=" $150 \quad$ "potential= " 3.293679248

$<$ me:PotentialPoint angle=" $180 \quad$ "potential= " $\quad 6.422936558$

$<$ me:PotentialPoint $\quad$ angle $=" \quad 210 \quad$ "potential= " 2.917500234

$<$ me:PotentialPoint

" potential="

angle $=" \quad 270 \quad$ "potential= " 3.433744422

"potential= "

6.413828699

angle $=" \quad 330 \quad$ "potential= " 2.809579056

$<$ me:PotentialPoint angle $=$

"potential="

1.989063047

$$
\begin{aligned}
& n /> \\
& " /> \\
& " /> \\
& " /> \\
& " /> \\
& " /> \\
& " /> \\
& " /> \\
& " /> \\
& " /> \\
& " /> \\
& " />
\end{aligned}
$$

$<$ /me:HinderedRotorPotential>

$<$ me:periodicity $>3</$ me:periodicity $>$

$<$ /me:ExtraDOSCMethod $>$

$<$ me:ExtraDOSCMethod xsi:type="HinderedRotorQM1D">

$<$ me:bondRef $>$ b $2</$ me:bondRef $>$

<me:HinderedRotorPotential format="numerical" units="kJ/mol" expansionSize="7" useSineTerms="yes" scale="1">

$<$ me:PotentialPoint $\quad$ angle=" $0 \quad 1.978445525$

$<$ me:PotentialPoin

angle $=" \quad 30$

"potential=

"potential="

" potential= "

"potential="

"potential= "

"potential= "

"potential="

"potential="

" potential= "

"potential="

"potential="

" potential="

1.978445525

6.721521546

20.83341076

13.52849261

4.067975955

0.008196811

4.067975955

13.52851099

22.24308635

angle=" 270

$<$ me:PotentialPoint angle $=" 300$

$<$ me:PotentialPoint angle $=$ " 330

$<$ me:PotentialPoint angle=" 360

6.721539925

2.62557E-06

1.978445525

"/>

$" />$

$" />$

$" />$

" />

$" />$

$" />$

$" />$

$" />$

$" />$

$" />$

$" />$

$<$ me:HinderedRotorPotential $>$

$<$ me:periodicity $>2</$ me:periodicity $>$

$<$ /me:ExtraDOSCMethod $>$

$<$ me:energyTransferModel xsi:type="me:ExponentialDown">

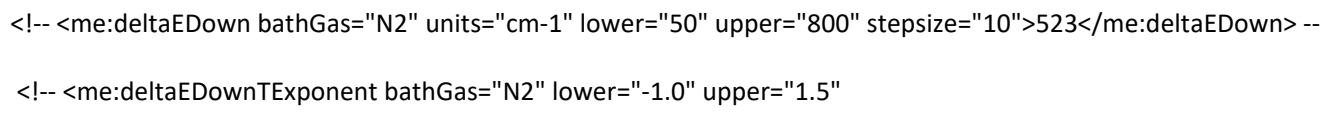




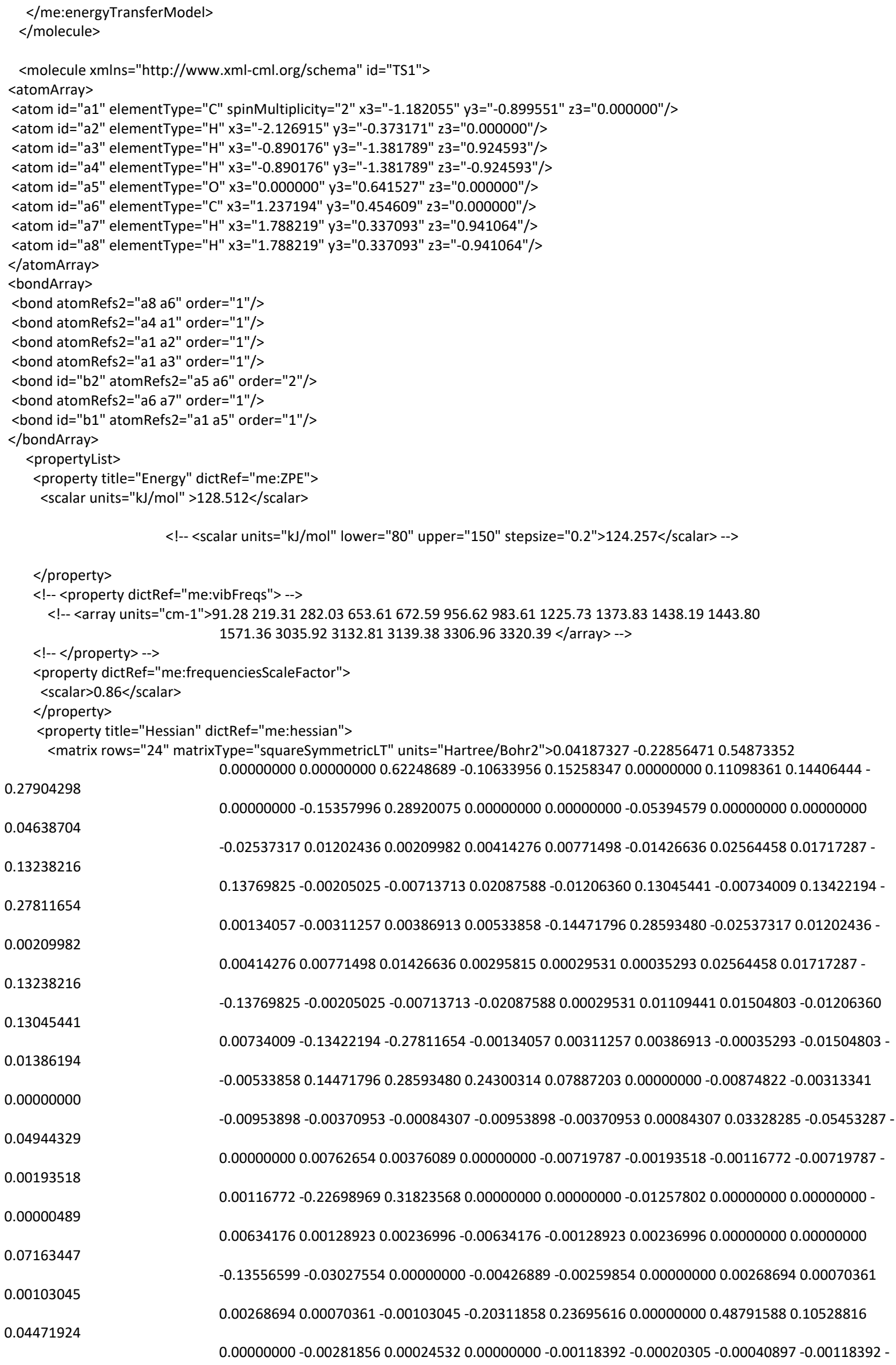




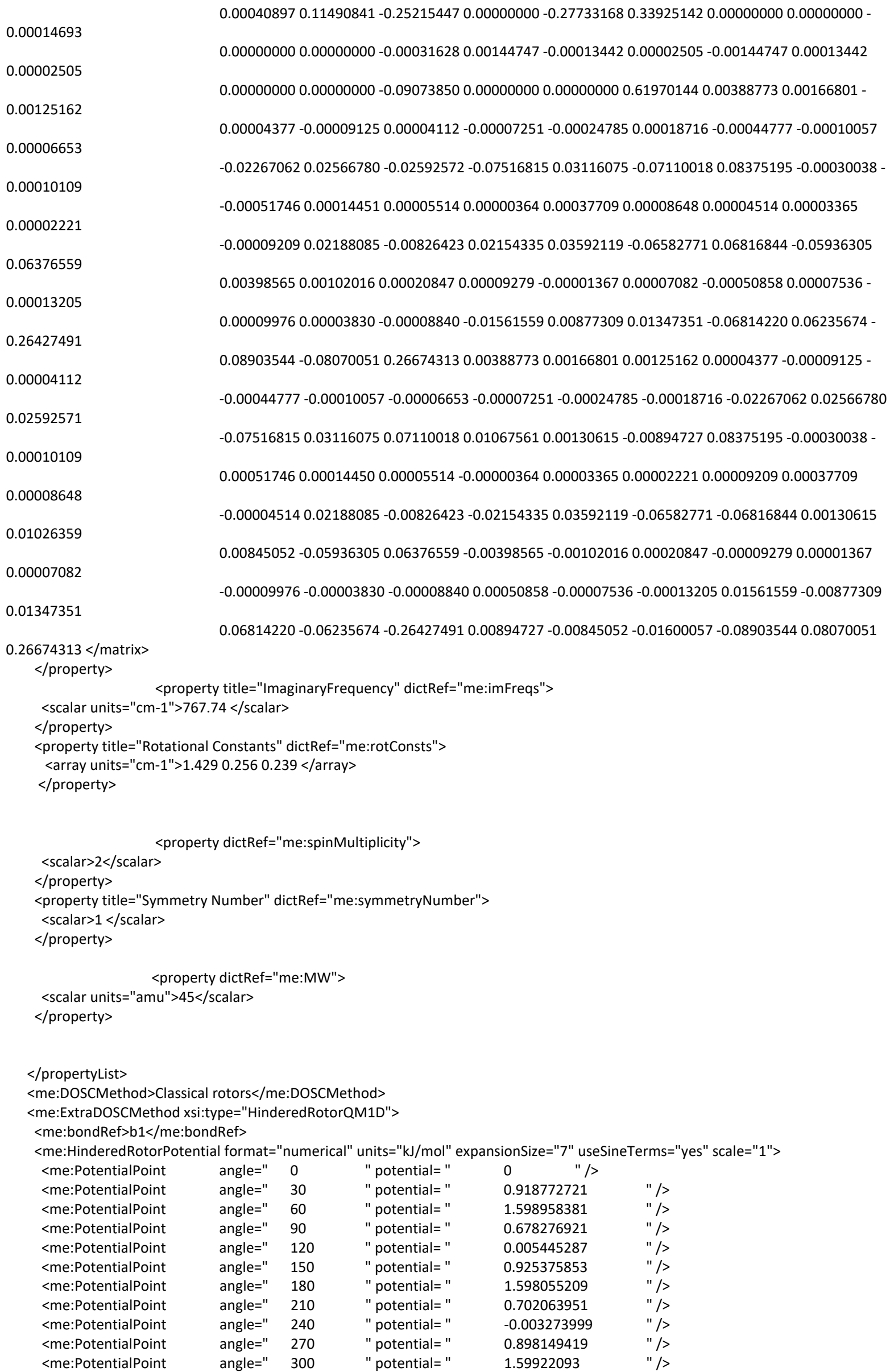




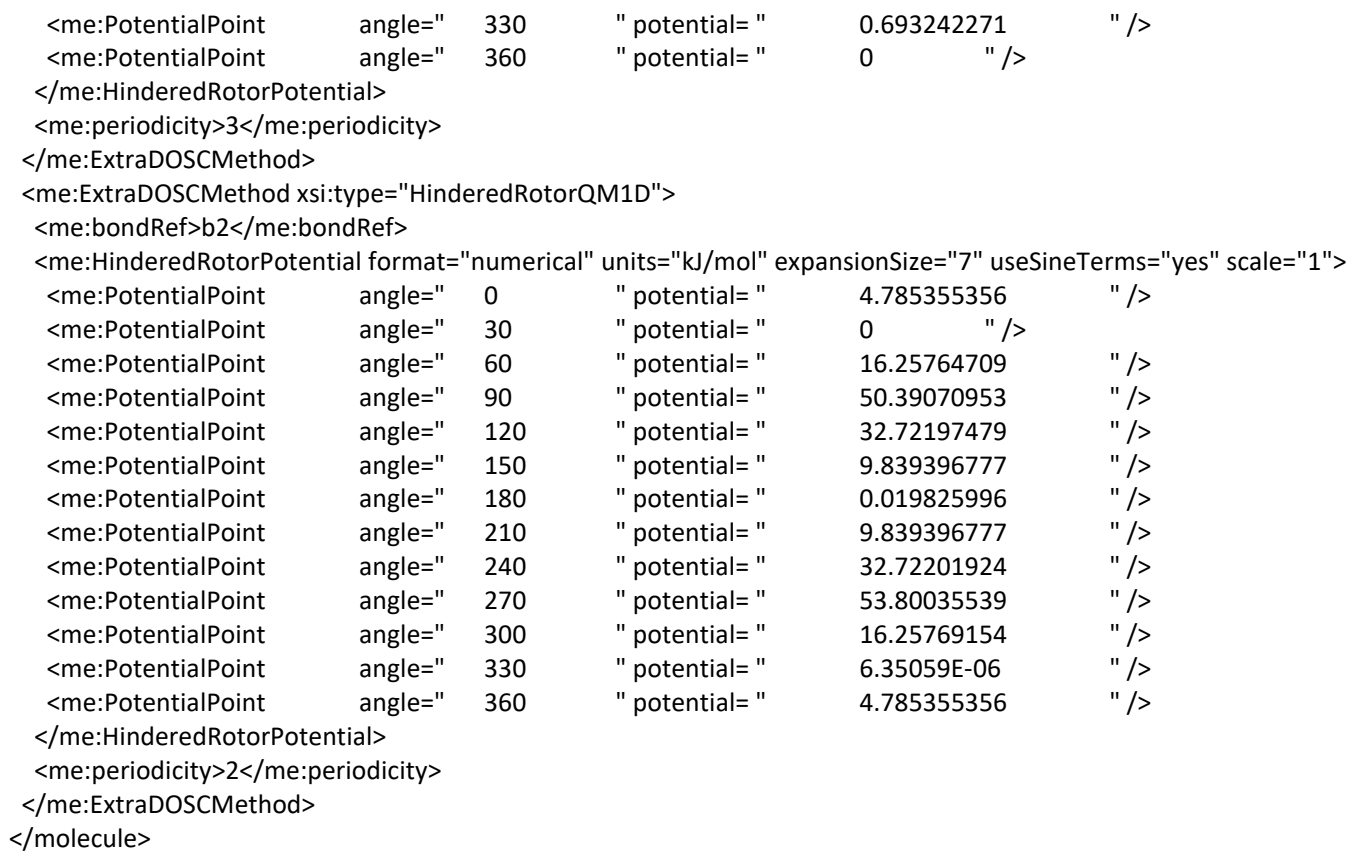

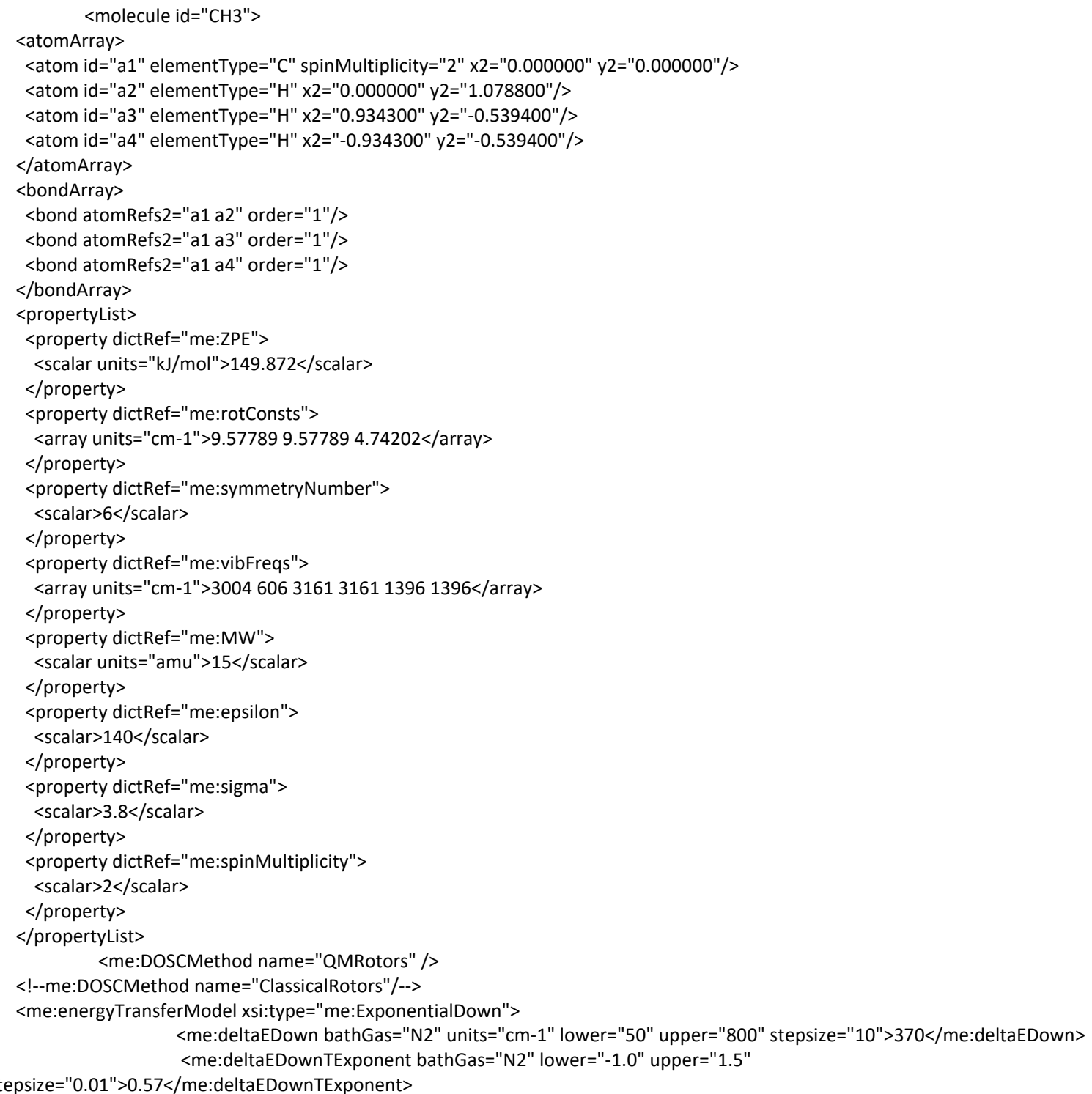


$<$ !-- <me:deltaEDown bathGas="N2" units="cm-1" > 250</me:deltaEDown> -->

$<$ !-- <me:deltaEDownTExponent bathGas="N2" >0.25</me:deltaEDownTExponent $>$-->

$<$ me:deltaEDown bathGas="He" units="cm-1" lower="50" upper="400" stepsize="10">192.511</me:deltaEDown> $<$ me:deltaEDownTExponent bathGas="He" lower="0.75" upper="1.5"

stepsize="0.01">0.75</me:deltaEDownTExponent $>$

stepsize="10">848</me:deltaEDown>

<me:deltaEDown bathGas="CH3OCH3" units="cm-1" lower="300" upper="4000"

upper="4.0" stepsize="0.01" >-.58</me:deltaEDownTExponent $>$

$<$ /me:energyTransferModel $>$

$</$ molecule $>$

<molecule xmlns="http://www.xml-cml.org/schema" id="CH2O">

$<$ atomArray>

<atom id="a1" elementType="O" x3="0.000000" y3="-0.674346" z3="0.000000"/>

<atom id="a2" elementType="C" x3="0.000000" y3="0.529185" z3="0.000000"/>

<atom id="a3" elementType="H" x3="-0.940850" y3="1.109829" z3="0.000000"/>

<atom id="a4" elementType="H" x3="0.940850" y3="1.109829" z3="0.000000"/>

$<$ /atomArray>

$<$ bondArray $>$

$<$ bond atomRefs2="a1 a2" order="2"/>

$<$ bond atomRefs2="a2 a3" order="1"/>

$<$ bond atomRefs2="a2 a4" order="1"/>

$<$ /bondArray $>$

$<$ propertyList>

<property title="Energy" dictRef="me:ZPE">

$<$ scalar units="kJ/mol">-105.379</scalar $>$

$</$ property $>$

<property dictRef $=$ "me:vibFreqs">

<array units="cm-1">27821746150011672843 1249</array>

$</$ property $>$

<property title="Rotational Constants" dictRef="me:rotConsts">

<array units="cm-1">9.4053 1.29530 1.13420</array>

$</$ property $>$

$<$ property title="Symmetry Number" dictRef="me:symmetryNumber" $>$

$<$ scalar $>2<$ /scalar $>$

$</$ property $>$

$<$ scalar $>1<$ scalar $>$

<property dictRef="me:spinMultiplicity">

$</$ property $>$

$<$ property dictRef="me:MW">

$<$ scalar units="amu" $>30<$ /scalar $>$

$</$ property $>$

$<$ property dictRef="me:epsilon">

$<$ scalar $>140<$ scalar $>$

$</$ property $>$

$<$ property dictRef="me:sigma">

$<$ scalar $>3.8<$ /scalar $>$

$</$ property $>$

$</$ propertyList $>$

<me:DOSCMethod name="QMRotors" />

<me:energyTransferModel xsi:type="me:ExponentialDown">

$<$ me:deltaEDown bathGas="N2" units="cm-1" lower="50" upper="800" stepsize="10">370</me:deltaEDown>

$<$ me:deltaEDownTExponent bathGas="N2" lower="-1.0" upper="1.5"

stepsize="0.01">0.57</me:deltaEDownTExponent $>$

$<$ !-- <me:deltaEDown bathGas="N2" units="cm-1" > 250</me:deltaEDown> -->

$<$ !-- <me:deltaEDownTExponent bathGas="N2" >0.25</me:deltaEDownTExponent $>->$

$<$ me:deltaEDown bathGas="He" units="cm-1" lower="50" upper="400" stepsize="10">192.511</me:deltaEDown>

$<$ me:deltaEDownTExponent bathGas="He" lower="0.75" upper="1.5"

stepsize="0.01">0.75</me:deltaEDownTExponent $>$

<me:deltaEDown bathGas="CH3OCH3" units="cm-1" lower="300" upper="4000"

stepsize $=" 10 ">848</$ me:deltaEDown $>$

$<$ me:deltaEDownTExponent bathGas=" $\mathrm{CH} 3 \mathrm{OCH} 3$ " referenceTemperature="521" lower="-1.0" upper="4.0" stepsize="0.01">-

$.58</$ me:deltaEDownTExponent>

$</$ me:energyTransferModel $>$

$<$ /molecule $>$ 


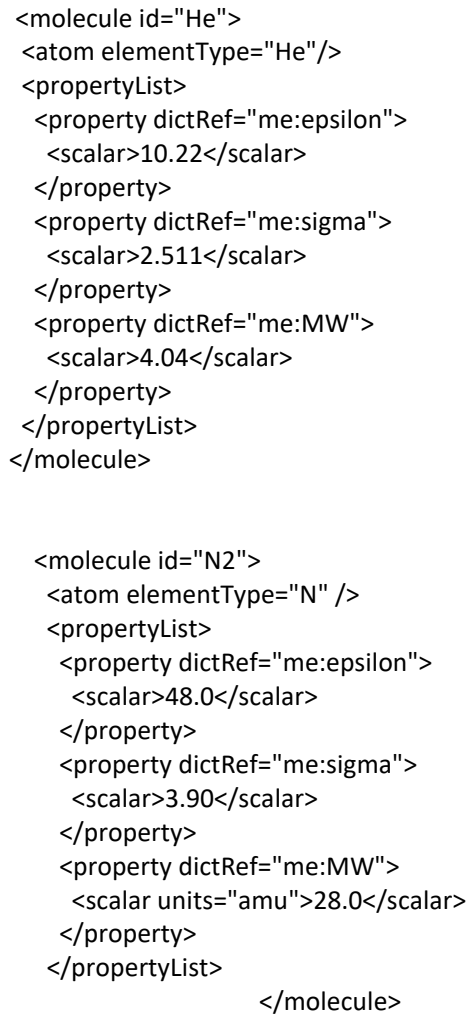

$<$ /molecule $>$

$<$ molecule xmlns="http://www.xml-cml.org/schema" id="CH3OCH3">

\section{$<$ atomArray>}

<atom id="a1" elementType="O" x3="0.000000" y3="0.000000" z3="0.601650"/> $<$ atom id="a2" elementType="C" x3="0.000000" y3="1.168190" z3="-0.200301"/> <atom id="a3" elementType="C" x3="0.000000" y3="-1.168190" z3="-0.200301"/> <atom id="a4" elementType="H" x3="0.000000" y3="2.020879" z3="0.477032"/> $<$ atom id="a5" elementType="H" x3="0.000000" y3="-2.020879" z3="0.477032"/> <atom id="a6" elementType="H" x3="0.890226" y3="1.216521" z3="-0.840911"/> <atom id="a7" elementType="H" x3="-0.890226" y3="1.216521" z3="-0.840911"/> <atom id="a8" elementType="H" x3="-0.890226" y3="-1.216521" z3="-0.840911"/> $<$ atom id="a9" elementType="H" x3="0.890226" y3="-1.216521" z3="-0.840911"/> $</$ atomArray $>$ 


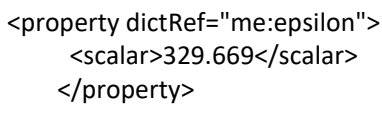

<property title="Symmetry Number" dictRef="me:symmetryNumber">

$<$ scalar $>2<$ /scalar $>$

<property dictRef="me:MW">

$<$ scalar units="amu" $>46.0</$ scalar $>$

$</$ property>

$<$ property $>$

$</$ propertyList $>$

$</$ molecule $>$

$<$ /moleculeList $>$

$<$ reactionList>

$<$ reaction id="R1">

$<$ reactant>

$<$ molecule ref="CH3OCH2" me:type="modelled"/>

$<$ reactant $>$

$<$ product $>$

$<$ molecule ref="CH2O" me:type="sink"/>

$</$ product $>$

$<$ product $>$

$<$ molecule ref="CH3" me:type="sink"/>

$</$ product $>$

$<$ me:transitionState $>$

$<$ molecule ref="TS1" me:type="transitionState"/>

$<$ me:transitionState $>$

$<!--<$ me:tunneling name="Eckart" $>>-->$

$<$ me:MCRCMethod>SimpleRRKM</me:MCRCMethod>

$</$ reaction $>$

$</$ reactionList $>$

$<$ me:conditions $>$

$<$ me:bathGas $>$ N2</me:bathGas $>$

$<$ me:PTs $>$

$<!--300 \mathrm{~s}-1$ propagated into the all errors and minus $200 \mathrm{~s}-1 \quad$-->

<me:PTpair me:units="PPCC" me:P=" 1.05147E17 " me:T=" 673 "><me:bathGas>He</me:bathGas><me:experimentalRate ref1="CH3OCH2" ref2="CH3OCH2" error="500"> 1772 $<$ /me:experimentalRate></me:PTpair > $<$ me:PTpair me:units="PPCC"
<

$\begin{array}{rl}<m e: P T p a i r & m e: \text { units="PPCC" } \\ & <\text { me:experimentalRate }\end{array}$ $<$ /me:experimentalRate>

$<$ me:PTpair me:units="PPCC" $<$ me:experimentalRate $<$ me:experimentalRate>

$<$ me:PTpair me:units="PPCC"

$<$ me:experimentalRate $<$ /me:experimentalRate> $\begin{array}{ll}<m e: P T p a i r & \text { me:units="PPCC" } \\ & <\text { me:experimentalRate }\end{array}$ $<$ /me:experimentalRate $>$

$<$ me:PTpair me:units="PPCC" $<$ me:experimentalRate $</$ me:experimentalRate>

<me:PTpair me:units="PPCC"

$<$ me:experimentalRate $<$ /me:experimentalRate

$<$ me:PTpair me:units="PPCC"

$<$ me:experimentalRate $<$ /me:experimentalRate $>$

$<m e: P T p a i r \quad$ me:units="PPCC" $<$ me:experimentalRate $<$ /me:experimentalRate $>$

$<$ me:PTpair me:units="PPCC" $<$ me:experimentalRate </me:experimentalRate>

$<$ me:PTpair me:units="PPCC" $<$ me:experimentalRate $<$ /me:experimentalRate $>$

$<m e: P T p a i r \quad m e: u n i t s=" P P C C "$ $<$ me:experimentalRate $<$ /me:experimentalRate me:P=" $1.05147 E 17$ me:T me:P=" $7.86525 E 17$ ref2="CH3OCH2" $<$ /me:PTpair> me:T=" ref1="CH3OCH2" ref2="CH3OCH2" me:P=" 7.86525E17 me:T=" ref1="CH3OCH2" ref2="CH3OCH2" me:P=" $1.57549 \mathrm{E} 18$ $\begin{array}{ll}\text { ref2="CH3OCH2" } & <\text { </me:PTpair> }\end{array}$ $\mathrm{me}: \mathrm{P}=" \quad 4.0141 \mathrm{E} 18$

me:P=" $113586 \mathrm{E} 17$ ref2="CH3OCH2" me: $P=" 1.13586 E 17 \quad$ " me:T=" ref1="CH3OCH2" ref2="CH3OCH2" me:P=" $1.13586 \mathrm{E} 17 \quad$ "<me:PTpair> me:T=" ref1="CH3OCH2" ref2="CH3OCH2" me:P=" 8.49649E17 $</$ me:PTpair> ref1="CH3OCH2" ref2="CH3OCH2"

me:P=" $1.93437 E 18 \quad$ " me:T=" $</$ me:PTpair> ref1="CH3OCH2" ref2="CH3OCH2"

me:P=" $1.70193 \mathrm{E} 18$ me:T=" $\begin{array}{ll}\text { ref } 1=" \mathrm{CH} 3 \mathrm{OCH} 2 " & \text { ref2="CH3OCH} 2 " \\ & </ \text { me:PTpair> }\end{array}$ me: $P=" \quad 4.33626 E 18$

ref1="CH3OCH2"
ref2="CH3OCH2" me:units="PPCC" $\quad 1.23498 E 17$

$<$ me:PTpair me:units="PPCC"
$<$ me:experimentalRate me: $\mathrm{P}="$ 1.23498E17 " me:T=" $</$ me:PTpair> ref1="CH3OCH2" ref2="CH3OCH2"

$$
673
$$
error= 673 error= 673 error= 673
error=

673 error

622 error= 622 error= 622 error=

622 error= 622 error 62 erro 573
error
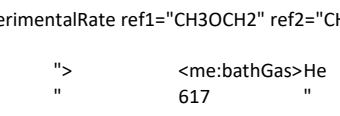

$<$ me:bathGas>

$</$ me:bathGas $>$

$\quad 477$

$<$ /me:bathGas>

$>\quad 13700$

$</$ me:bathGas>

$>\quad 23275$

$<$ me:bathGas>

$>951$

$<$ /me:bathGas>

\begin{tabular}{l}
$>\quad 2747$ \\
\hline
\end{tabular}

$</$ me:bathGas>

$>\quad 1948$

$<$ me:bathGas>

$>\quad 3347$

$<$ me:bathGas>

$</$ me:bathGas $>$ $37.69215</$ me:experimentalRate 


$\begin{array}{cl}\text { <me:PTpair } & \text { me:units="PPCC" } \\ & <\text { me:experimentalRate } \\ & <\text { me:experimentalRate> } \\ \text { me:units="PPCC" } & \\ & <\text { me:experimentalRate } \\ & <\text { /me:experimentalRate> } \\ \text { <me:PTpair } & \text { me:units="PPCC" } \\ & <\text { me:experimentalRate } \\ & <\text { /me:experimentalRate> }\end{array}$

\begin{tabular}{|c|c|}
\hline \multirow{2}{*}{\multicolumn{2}{|c|}{$\begin{array}{l}\text { me:P=" } 9.2379 E 17 \\
\text { ref1="CH3OCH2" }\end{array}$}} \\
\hline & \\
\hline me:P=" & 2.10317E18 \\
\hline \multicolumn{2}{|c|}{ ref1="CH3OCH2" } \\
\hline me:P=" & 1.85044E18 \\
\hline ref1="Cl & H2" \\
\hline
\end{tabular}

573
error=
573
error=
573
error=

ref2="CH3OCH2"

<me:bathGas> He
360
<me:bathGas> He
360
<me:bathGas> He
$323 \quad "$

$>\quad 791$

$</$ me:bathGas>

$</$ me:bathGas>

$>\quad 396$

\begin{tabular}{|c|c|c|c|c|}
\hline \multicolumn{5}{|c|}{ <!--Loucks and Laidler--> } \\
\hline \multirow[t]{3}{*}{ <me:PTpair } & me:units="PPCC" & $5.52551 \mathrm{E} 16$ & \multirow{2}{*}{$\begin{array}{l}\text { " me:T=" } \\
\text { ref2="CH3OCH2" }\end{array}$} & 573 \\
\hline & $<$ me:experimentalRate & ref1="CH3OCH2" & & error $=$ \\
\hline & $155.68109</$ me:experim & $<$ /me:experimentalRate $>$ & </me:PTpair> & \\
\hline \multirow[t]{3}{*}{ <me:PTpair } & me:units="PPCC" & 9.12794E16 & me:T=" & 573 \\
\hline & $<$ me:experimentalRate & ref1="CH3OCH2" & ref2="CH3OCH2" & error $=$ \\
\hline & $199.63462</$ me:experim & nentalRate> & $<$ /me:PTpair> & \\
\hline <me:PTpair & me:units="PPCC" & 1.20607E17 & me:T=" & 573 \\
\hline & $<$ me:experimentalRate & ref1="CH3OCH2" & ref2="CH3OCH2" & error $=$ \\
\hline & $179.57086</$ me:experim & nentalRate> & $<$ /me:PTpair> & \\
\hline$<$ me:PTpair & me:units="PPCC" & 2.48526E17 & me:T=" & 573 \\
\hline & $<$ me:experimentalRate & ref1="CH3OCH2" & ref2="CH3OCH2" & error $=$ \\
\hline & $238.36136</$ me:experim & nentalRate> & $<$ <me:PTpair> & \\
\hline <me:PTpair & me:units="PPCC" & 3.47835E17 & me:T=" & 573 \\
\hline & $<$ me:experimentalRate & ref1="CH3OCH2" & ref2="CH3OCH2" & error $=$ \\
\hline & $264.99389</$ me:experin & nentalRate> & $<$ /me:PTpair > & \\
\hline <me:PTpair & me:units="PPCC" & 5.42466E17 & me:T=" & 573 \\
\hline & $<$ me:experimentalRate & ref1="CH3OCH2" & ref2="CH3OCH2" & error= \\
\hline & $391.05321</$ me:experin & nentalRate> & $<$ /me:PTpair> & \\
\hline$<$ me:PTpair & me:units="PPCC" & 8.47955E17 & me:T=" & 573 \\
\hline & $<$ me:experimentalRate & ref1="CH3OCH2" & ref2="CH3OCH2" & error $=$ \\
\hline & $641.5579</$ me:experin & nentalRate> & $<$ <me:PTpair> & \\
\hline <me:PTpair & me:units="PPCC" & $9.4705 \mathrm{E} 17$ & me:T=" & 573 \\
\hline & $<$ me:experimentalRate & ref1="CH3OCH2" & ref2="CH3OCH2" & error $=$ \\
\hline & $483.32121</$ me:experim & nentalRate> & $<$ /me:PTpair> & \\
\hline <me:PTpair & me:units="PPCC" & $1.65338 \mathrm{E} 18$ & me:T=" & 573 \\
\hline & $<$ me:experimentalRate & ref1="CH3OCH2" & ref2="CH3OCH2" & error $=$ \\
\hline & $665.63455</$ me:experim & nentalRate> & $<$ /me:PTpair> & \\
\hline <me:PTpair & me:units="PPCC" & 4.03063E18 & me:T=" & 573 \\
\hline & $<$ me:experimentalRate & ref1="CH3OCH2" & ref2="CH3OCH2" & error $=$ \\
\hline & $714.88446</$ me:experim & nentalRate> & $<$ /me:PTpair> & \\
\hline <me:PTpair & me:units="PPCC" & 6.16208 E16 & me:T=" & 543 \\
\hline & $<$ me:experimentalRate & ref1="CH3OCH2" & ref2="CH3OCH2" & error $=$ \\
\hline & $62.5794</$ me:experim & nentalRate> & $<$ /me:PTpair> & \\
\hline <me:PTpair & me:units="PPCC" & 9.63224E16 & me:T=" & 543 \\
\hline & $<$ me:experimentalRate & ref1="CH3OCH2" & ref2="CH3OCH2" & error $=$ \\
\hline & $72.18241</$ me:experin & nentalRate> & $<$ /me:PTpair> & \\
\hline <me:PTpair & me:units="PPCC" & $1.20428 \mathrm{E} 17$ & me:T=" & 543 \\
\hline & $<$ me:experimentalRate & ref1="CH3OCH2" & ref2="CH3OCH2" & error $=$ \\
\hline & $80.24748</$ me:experin & nentalRate> & $<$ /me:PTpair> & \\
\hline <me:PTpair & me:units="PPCC" & 2.22192E17 & me:T=" & 543 \\
\hline & $<$ me:experimentalRate & ref1="CH3OCH2" & ref2="CH3OCH2" & error $=$ \\
\hline & $</$ me:experim & nentalRate> & $<$ /me:PTpair> & \\
\hline <me:PTpair & me:units="PPCC" & 3.87907E17 & me:T=" & 543 \\
\hline & $<$ me:experimentalRate & ref1="CH3OCH2" & ref2="CH3OCH2" & error $=$ \\
\hline & $110.2634</$ me:experim & nentalRate> & $<$ /me:PTpair> & \\
\hline$<m e:$ PTpair & me:units="PPCC" & $6.77216 \mathrm{E} 17$ & me:T=" & 543 \\
\hline & $<$ me:experimentalRate & ref1="CH3OCH2" & ref2="CH3OCH2" & error $=$ \\
\hline & $118.42172</$ me:experim & nentalRate> & $<$ /me:PTpair> & \\
\hline <me:PTpair & me:units="PPCC" & 7.15694E17 & me:T=" & 543 \\
\hline & $<$ me:experimentalRate & ref1="CH3OCH2" & ref2="CH3OCH2" & error $=$ \\
\hline & $146.36303</$ me:experin & nentalRate> & $<$ /me:PTpair> & \\
\hline <me:PTpair & me:units="PPCC" & 1.11616E18 & me:T=" & 543 \\
\hline & $<$ me:experimentalRate & ref1="CH3OCH2" & ref2="CH3OCH2" & error $=$ \\
\hline & $174.75571</$ me:experin & nentalRate> & $<$ /me:PTpair > & \\
\hline <me:PTpair & me:units="PPCC" & 1.65093E18 & me:T=" & 543 \\
\hline & $<$ me:experimentalRate & ref1="CH3OCH2" & ref2="CH3OCH2" & error $=$ \\
\hline & $208.65624</$ me:experim & nentalRate> & </me:PTpair> & \\
\hline$<$ me:PTpair & me:units="PPCC" & $2.57471 \mathrm{E} 18$ & me: $T="$ & 543 \\
\hline & $<$ me:experimentalRate & ref1="CH3OCH2" & ref2="CH3OCH2" & error $=$ \\
\hline & $287.36335</$ me:experim & nentalRate> & </me:PTpair> & \\
\hline$<$ me:PTpair & me:units="PPCC" & $2.88222 \mathrm{E} 18$ & me:T=" & 543 \\
\hline & $<$ me:experimentalRate & ref1="CH3OCH2" & ref2="CH3OCH2" & error= \\
\hline & $330.69786<$ /me:experin & nentalRate> & $<$ /me:PTpair> & \\
\hline <me:PTpair & me:units="PPCC" & $3.04598 \mathrm{E} 18$ & me:T=" & 543 \\
\hline & $<$ me:experimentalRate & ref1="CH3OCH2" & ref2="CH3OCH2" & error $=$ \\
\hline & $257.88815<$ /me:experin & nentalRate> & </me:PTpair> & \\
\hline <me:PTpair & me:units="PPCC" & 5.31774E18 & me:T=" & 543 \\
\hline & $<$ me:experimentalRate & ref1="CH3OCH2" & ref2="CH3OCH2" & error $=$ \\
\hline & $408.72517<$ /me:experin & nentalRate> & </me:PTpair> & \\
\hline <me:PTpair & me:units="PPCC" & 6.80283E16 & me:T=" & 521 \\
\hline & $<m e:$ experimentalRate & ref1="CH3OCH2" & ref2="CH3OCH2" & error $=$ \\
\hline & $25.10818</$ me:experim & nentalRate> & </me:PTpair> & \\
\hline <me:PTpair & me:units="PPCC" & $1.12122 \mathrm{E} 17$ & me:T=" & 521 \\
\hline & $<$ me:experimentalRate & ref1="CH3OCH2" & ref2="CH3OCH2" & error $=$ \\
\hline & $25.99053</$ me:experim & nentalRate> & </me:PTpair> & \\
\hline <me:PTpair & me:units="PPCC" & 1.48146E17 & me:T=" & 521 \\
\hline & $<$ me:experimentalRate & ref1="CH3OCH2" & ref2="CH3OCH2" & error $=$ \\
\hline & $28.96111</$ me:experim & nentalRate> & </me:PTpair> & \\
\hline <me:PTpair & me:units="PPCC" & 1.75263E17 & me:T=" & 521 \\
\hline & $<$ me:experimentalRate & ref1="CH3OCH2" & ref2="CH3OCH2" & error $=$ \\
\hline & $</$ me:experim & nentalRate> & </me:PTpair> & \\
\hline
\end{tabular}
31.13622

$<$ me:bath Gas $>\mathrm{CH} 3 \mathrm{OCH} 3$ $39.92692 \quad "$

$<$ me:bathGas $>\mathrm{CH} 3 \mathrm{OCH} 3$ $35.91417 \quad "$

$<$ me:bathGas>CH3OCH3 47.67227

$<$ me:bathGas>CH3OCH3 52.99878

$<$ me:bathGas>CH3OCH3 $78.21064 \quad "$

$<$ me:bathGas $>\mathrm{CH} 3 \mathrm{OCH} 3$ 128.31158

<me:bathGas>CH3OCH3 96.66424

$<$ me:bathGas>CH3OCH3 $133.12691 \quad "$

$<$ me:bathGas>CH3OCH3 142.97689 " >

$<$ me:bathGas>CH3OCH3 </me:bathGas > 12.51588

$<$ me:bathGas $>\mathrm{CH} 3 \mathrm{OCH} 3$ $14.43648 \quad "$

$<$ me:bathGas $>\mathrm{CH} 3 \mathrm{OCH} 3$ $16.0495 \quad$ " >

$<$ me:bathGas $>\mathrm{CH} 3 \mathrm{OCH} 3 \quad<$ me:bathGas $>$ 19.83633

$<$ me:bathGas $>\mathrm{CH} 3 \mathrm{OCH} 3$ $22.05268 \quad "$

$<$ me:bathGas $>\mathrm{CH} 3 \mathrm{OCH} 3$ 23.68434

<me:bathGas>CH3OCH3 29.27261

$<$ me:bath Gas $>\mathrm{CH} 3 \mathrm{OCH} 3$ 34.95114

$<$ me:bathGas>CH3OCH3 41.73125

<me:bathGas>CH3OCH3 57.47267

$<$ me:bathGas>CH3OCH3 $66.13957 "$

$<$ me:bathGas>CH3OCH3 $51.57763>$

$<$ me:bathGas>CH3OCH3 </me:bathGas> 81.74503

$<$ me:bathGas $>\mathrm{CH} 3 \mathrm{OCH} 3$ $5.02164 ">$

<me:bathGas>CH3OCH3 </me:bathGas> 5.19811

$<$ me:bathGas>CH3OCH3 5.79222

$<$ me:bathGas> $\mathrm{CH} 3 \mathrm{OCH} 3$ 6.20648
me:bathGas>

$<$ me:bathGas >

me:bathGas $>$

me:bathGas>

$<$ me:bathGas >

me:bathGas $>$

/me:bathGas>

$<$ me:bathGas >

me:bathGas>

me:bathGas>

$<$ /me:bathGas >

me:bathGas>

$<$ /me:bathGas>

$<$ /me:bathGas>

$<$ me:bathGas $>$

$<$ me:bathGas>

$<$ /me:bathGas>

$</$ me:bathGas $>$

$<$ me:bathGas

$\langle$ me.bathGas

$\langle$ mebathGas

$<$ /me:bathGas>

$</$ me:bathGas $>$

$>$

$<$ me:bathGas> 


\begin{tabular}{|c|c|c|c|c|c|c|c|}
\hline$<$ me:PTpair & me:units="PPCC" & 2.31574E17 & me:T=" & 521 & "> & $<$ me:bathGas $>\mathrm{CH} 3 \mathrm{OCH} 3$ & $</$ me:bathGas $>$ \\
\hline & $<$ me:experimentalRate & ref1="CH3OCH2" & ref2="CH3OCH2" & error= & " & 7.15888 & $>$ \\
\hline & $35.79441</$ me:experir & nentalRate> & </me:PTpair> & & & & \\
\hline <me:PTpair & me:units="PPCC" & 3.41735E17 & me:T=" & 521 & "> & $<$ me:bathGas $>\mathrm{CH} 3 \mathrm{OCH} 3$ & $</$ me:bathGas $>$ \\
\hline & $<$ cme:experimentalRate & ref1="CH3OCH2" & ref2="CH3OCH2" & error= & $"$ & $8.23844 \quad " \quad$ & $>$ \\
\hline & $41.19222</$ me:experir & nentalRate> & $<$ <me:PTpair> & & & & \\
\hline <me:PTpair & me:units="PPCC" & $5.04301 \mathrm{E} 17$ & me:T=" & 521 & "> & $<$ me:bathGas $>\mathrm{CH} 3 \mathrm{OCH} 3$ & $</$ me:bathGas $>$ \\
\hline & $<$ me:experimentalRate & ref1="CH3OCH2" & ref2="CH3OCH2" & error= & $"$ & $8.54762 \quad " \quad$ & $>$ \\
\hline & $</$ me:experir & nentalRate> & </me:PTpair> & & & & \\
\hline <me:PTpair & me:units="PPCC" & 5.96609E17 & me:T=" & 521 & "> & $<$ me:bathGas $>\mathrm{CH} 3 \mathrm{OCH} 3$ & $</$ me:bathGas $>$ \\
\hline & $<$ me:experimentalRate & ref1="CH3OCH2" & ref2="CH3OCH2" & error $=$ & $"$ & $9.18005 \quad$ " & $>$ \\
\hline & $</$ me:experir & nentalRate> & $<$ <me:PTpair> & & & & \\
\hline <me:PTpair & me:units="PPCC" & 7.45915E17 & me:T=" & 521 & "> & $<$ me:bathGas $>\mathrm{CH} 3 \mathrm{OCH} 3$ & $<$ /me:bathGas $>$ \\
\hline & $<$ me:experimentalRate & ref1="CH3OCH2" & ref2="CH3OCH2" & error= & $"$ & $11.34606 \quad "$ & $>$ \\
\hline & $</$ me:experir & nentalRate> & $<$ <me:PTpair> & & & & \\
\hline <me:PTpair & me:units="PPCC" & 1.16329E18 & me:T=" & 521 & "> & $<$ me:bathGas $>\mathrm{CH} 3 \mathrm{OCH} 3$ & $<$ /me:bathGas $>$ \\
\hline & $<$ me:experimentalRate & ref1="CH3OCH2" & ref2="CH3OCH2" & error= & $"$ & $13.05705 \quad "$ & $>$ \\
\hline & $</$ me:experir & nentalRate> & $<$ /me:PTpair> & & & & \\
\hline <me:PTpair & me:units="PPCC" & $1.8184 \mathrm{E} 18$ & me:T=" & 521 & "> & $<$ me:bathGas $>\mathrm{CH} 3 \mathrm{OCH} 3$ & $<$ /me:bathGas $>$ \\
\hline & $<$ me:experimentalRate & ref1="CH3OCH2" & ref2="CH3OCH2" & error= & $"$ & $15.0607 \quad "$ & $>$ \\
\hline & $</$ me:experir & nentalRate> & $<$ <me:PTpair> & & & & \\
\hline <me:PTpair & me:units="PPCC" & 3.5456E18 & me:T=" & 521 & "> & $<$ me:bathGas $>\mathrm{CH} 3 \mathrm{OCH} 3$ & $</$ me:bathGas $>$ \\
\hline & $<$ me:experimentalRate & ref1="CH3OCH2" & ref2="CH3OCH2" & error= & $"$ & $19.3128 \quad "$ & $>$ \\
\hline & $96.56398</$ me:experir & nentalRate> & $<$ /me:PTpair> & & & & \\
\hline <me:PTpair & me:units="PPCC" & $6.54168 \mathrm{E} 18$ & me:T=" & 521 & "> & $<$ me:bathGas $>\mathrm{CH} 3 \mathrm{OCH} 3$ & $</$ me:bathGas $>$ \\
\hline & $<$ me:experimentalRate & ref1="CH3OCH2" & ref2="CH3OCH2" & error= & $"$ & $21.47065 \quad "$ & $>$ \\
\hline & 107.35324</me:experir & nentalRate> & </me:PTpair> & & & & \\
\hline <me:PTpair & me:units="PPCC" & 1.20973E19 & me:T=" & 521 & "> & $<$ me:bathGas $>\mathrm{CH} 3 \mathrm{OCH} 3$ & $</$ me:bathGas $>$ \\
\hline & $<$ me:experimentalRate & ref1="CH3OCH2" & ref2="CH3OCH2" & error= & " & 26.53659 & $>$ \\
\hline & $132.68297</$ me:experir & nentalRate> & $<$ /me:PTpair> & & & & \\
\hline <me:PTpair & me:units="PPCC" & 6.01586 E16 & me:T=" & 498 & "> & $<$ me:bathGas $>\mathrm{CH} 3 \mathrm{OCH} 3$ & $<$ /me:bathGas $>$ \\
\hline & $<$ me:experimentalRate & ref1="CH3OCH2" & ref2="CH3OCH2" & error= & " & 1.94851 & $>$ \\
\hline & $9.74255</$ me:experir & nentalRate> & $<$ <me:PTpair> & & & & \\
\hline <me:PTpair & me:units="PPCC" & $1.05026 \mathrm{E} 17$ & me:T=" & 498 & "> & $<$ me:bathGas $>\mathrm{CH} 3 \mathrm{OCH} 3$ & $<$ /me:bathGas $>$ \\
\hline & $<$ me:experimentalRate & ref1="CH3OCH2" & ref2="CH3OCH2" & error= & $"$ & 2.08787 & $>$ \\
\hline & $10.43933</$ me:experir & nentalRate> & $<$ /me:PTpair> & & & & \\
\hline <me:PTpair & me:units="PPCC" & 1.83357E17 & me:T=" & 498 & "> & $<$ me:bathGas $>\mathrm{CH} 3 \mathrm{OCH} 3$ & $</$ me:bathGas $>$ \\
\hline & <me:experimentalRate & ref1="CH3OCH2" & ref2="CH3OCH2" & error= & $"$ & 2.49289 & $>$ \\
\hline & $12.46443<$ /me:experir & nentalRate> & $<$ <me:PTpair> & & & & \\
\hline$<$ eme:PTpair & me:units="PPCC" & 2.85955E17 & me:T=" & 498 & "> & $<$ me:bathGas $>\mathrm{CH} 3 \mathrm{OCH} 3$ & $</$ me:bathGas $>$ \\
\hline & $<$ me:experimentalRate & ref1="CH3OCH2" & ref2="CH3OCH2" & error= & $"$ & 2.97648 & $>$ \\
\hline & $14.88238<$ <me:experir & nentalRate> & $<$ <me:PTpair> & & & & \\
\hline <me:PTpair & me:units="PPCC" & 4.99227E17 & me:T=" & 498 & "> & $<$ me:bathGas $>\mathrm{CH} 3 \mathrm{OCH} 3$ & $</$ me:bathGas $>$ \\
\hline & <me:experimentalRate & ref1="CH3OCH2" & ref2="CH3OCH2" & error= & $"$ & 3.81683 & $>$ \\
\hline & $19.08413</$ me:experir & nentalRate> & $<$ /me:PTpair> & & & & \\
\hline <me:PTpair & me:units="PPCC" & $6.97105 E 17$ & me:T=" & 498 & "> & $<$ me:bathGas $>\mathrm{CH} 3 \mathrm{OCH} 3$ & $</$ me:bathGas $>$ \\
\hline & $<$ me:experimentalRate & ref1="CH3OCH2" & ref2="CH3OCH2" & error= & " & 4.24329 & $>$ \\
\hline & $21.21644</$ me:experir & nentalRate> & $<$ /me:PTpair> & & & & \\
\hline <me:PTpair & me:units="PPCC" & 8.24704E17 & me:T=" & 498 & "> & $<$ me:bathGas $>\mathrm{CH} 3 \mathrm{OCH} 3$ & $</$ me:bathGas $>$ \\
\hline & $<$ me:experimentalRate & ref1="CH3OCH2" & ref2="CH3OCH2" & error= & " & 4.24329 & $>$ \\
\hline & $21.21644<$ <me:experir & nentalRate> & </me:PTpair> & & & & \\
\hline <me:PTpair & me:units="PPCC" & 1.15159E18 & me:T=" & 498 & "> & $<$ me:bathGas $>\mathrm{CH} 3 \mathrm{OCH} 3$ & $</$ me:bathGas $>$ \\
\hline & $<$ me:experimentalRate & ref1="CH3OCH2" & ref2="CH3OCH2" & error= & $"$ & 4.7174 & $>$ \\
\hline & $</$ me:experir & nentalRate> & $<$ /me:PTpair> & & & & \\
\hline$<$ me:PTpair & me:units="PPCC" & 1.90238 E18 & me:T=" & 498 & "> & $<$ me:bathGas $>\mathrm{CH} 3 \mathrm{OCH} 3$ & $</$ me:bathGas $>$ \\
\hline & $<$ me:experimentalRate & ref1="CH3OCH2" & ref2="CH3OCH2" & error= & $"$ & 5.4413 & $>$ \\
\hline & $</$ me:experir & nentalRate> & </me:PTpair> & & & & \\
\hline$<$ eme:PTpair & me:units="PPCC" & $3.14266 \mathrm{E} 18$ & me:T=" & 498 & "> & $<$ me:bathGas $>\mathrm{CH} 3 \mathrm{OCH} 3$ & $</$ me:bathGas $>$ \\
\hline & $<$ me:experimentalRate & ref1="CH3OCH2" & ref2="CH3OCH2" & error= & $"$ & 6.26185 & $>$ \\
\hline & $</$ me:experir & nentalRate> & </me:PTpair> & & & & \\
\hline <me:PTpair & me:units="PPCC" & 4.63765E18 & me:T=" & 498 & "> & $<$ me:bathGas $>\mathrm{CH} 3 \mathrm{OCH} 3$ & $</$ me:bathGas $>$ \\
\hline & $<$ me:experimentalRate & ref1="CH3OCH2" & ref2="CH3OCH2" & error= & " & 7.47657 & $>$ \\
\hline & $</$ me:experir & nentalRate> & </me:PTpair> & & & & \\
\hline <me:PTpair & me:units="PPCC" & $8.09651 \mathrm{E} 18$ & me:T=" & 498 & "> & $<$ me:bathGas $>\mathrm{CH} 3 \mathrm{OCH} 3$ & $</$ me:bathGas $>$ \\
\hline & $<$ me:experimentalRate & ref1="CH3OCH2" & ref2="CH3OCH2" & error= & " & 9.58744 & $>$ \\
\hline & $</$ me:experir & nentalRate> & </me:PTpair> & & & & \\
\hline <me:PTpair & me:units="PPCC" & 7.91892E16 & me:T=" & 473 & "> & $<$ me:bathGas $>\mathrm{CH} 3 \mathrm{OCH} 3$ & $</$ me:bathGas $>$ \\
\hline & $<$ me:experimentalRate & ref1="CH3OCH2" & ref2="CH3OCH2" & error= & " & 0.67971 & $>$ \\
\hline & $</$ me:experir & nentalRate> & </me:PTpair> & & & & \\
\hline$<$ me:PTpair & me:units="PPCC" & 9.87794E16 & me:T=" & 473 & "> & $<$ me:bathGas $>\mathrm{CH} 3 \mathrm{OCH} 3$ & $<$ /me:bathGas $>$ \\
\hline & $<$ me:experimentalRate & ref1="CH3OCH2" & ref2="CH3OCH2" & error= & " & 0.65663 & $>$ \\
\hline & $</$ me:experir & nentalRate> & </me:PTpair> & & & & \\
\hline$<$ me:PTpair & me:units="PPCC" & 1.10577E17 & me:T=" & 473 & "> & $<$ me:bathGas $>\mathrm{CH} 3 \mathrm{OCH} 3$ & $</$ me:bathGas $>$ \\
\hline & $<$ me:experimentalRate & ref1="CH3OCH2" & ref2="CH3OCH2" & error= & " & 0.75565 & $>$ \\
\hline & $</$ me:experir & nentalRate> & </me:PTpair> & & & & \\
\hline <me:PTpair & me:units="PPCC" & 2.04017E17 & me:T=" & 473 & "> & $<$ me:bathGas $>\mathrm{CH} 3 \mathrm{OCH} 3$ & $</$ me:bathGas $>$ \\
\hline & $\begin{array}{l}<\text { <me:experimentalRate } \\
</ \text { me:experimentalRate }>\end{array}$ & ref1="CH3OCH2" & $\begin{array}{l}\text { ref2="CH3OCH2" } \\
</ \text { me:PTpair> }\end{array}$ & error $=$ & " & 0.81156 & $>\quad 4.0578$ \\
\hline <me:PTpair & me:units="PPCC" & $2.55074 \mathrm{E} 17$ & me:T=" & 473 & "> & $<$ me:bathGas $>\mathrm{CH} 3 \mathrm{OCH} 3$ & $</$ me:bathGas $>$ \\
\hline & $<$ me:experimentalRate & ref1="CH3OCH2" & ref2="CH3OCH2" & error= & " & 0.96899 & $>$ \\
\hline & $4.84496 \quad</$ me:experir & mentalRate> & </me:PTpair> & & & & \\
\hline <me:PTpair & me:units="PPCC" & 5.55477E17 & me:T=" & 473 & "> & $<$ me:bathGas $>\mathrm{CH} 3 \mathrm{OCH} 3$ & $</$ me:bathGas $>$ \\
\hline & $<$ me:experimentalRate & ref1="CH3OCH2" & ref2="CH3OCH2" & error= & " & 0.87161 & $>$ \\
\hline & $4.35803</$ me:experir & nentalRate> & </me:PTpair> & & & & \\
\hline <me:PTpair & me:units="PPCC" & 4.97355E 17 & me:T=" & 473 & "> & $<$ me:bathGas $>\mathrm{CH} 3 \mathrm{OCH} 3$ & $<$ /me:bathGas > \\
\hline & $<$ me:experimentalRate & ref1="CH3OCH2" & ref2="CH3OCH2" & error= & " & $1.04069 \quad "$ & $>$ \\
\hline & $5.20344</$ me:experir & nentalRate> & </me:PTpair> & & & & \\
\hline <me:PTpair & me:units="PPCC" & 7.77439EE17 & me:T=" & 473 & "> & $<$ me:bathGas $>\mathrm{CH} 3 \mathrm{OCH} 3$ & $</$ me:bathGas $>$ \\
\hline & $\begin{array}{l}<\text { <e:experimentalRate } \\
</ \text { me:experimentalRate }>\end{array}$ & ref1="CH3OCH2" & $\begin{array}{l}\text { ref2="CH3OCH2" } \\
<\text { /me:PTpair> }\end{array}$ & error= & " & 1.07726 & 5.3863 \\
\hline <me:PTpair & me:units="PPCC" & $8.21611 \mathrm{E} 17$ & me:T=" & 473 & "> & $<$ me:bathGas $>\mathrm{CH} 3 \mathrm{OCH} 3$ & $</$ me:bathGas $>$ \\
\hline & $\begin{array}{l}<\text { <me:experimentalRate } \\
</ \text { me:experimentalRate }>\end{array}$ & ref1="CH3OCH2" & $\begin{array}{l}\text { ref2="CH3OCH2" } \\
</ \text { me:PTpair> }\end{array}$ & error= & " & 1.07726 & 5.3863 \\
\hline
\end{tabular}




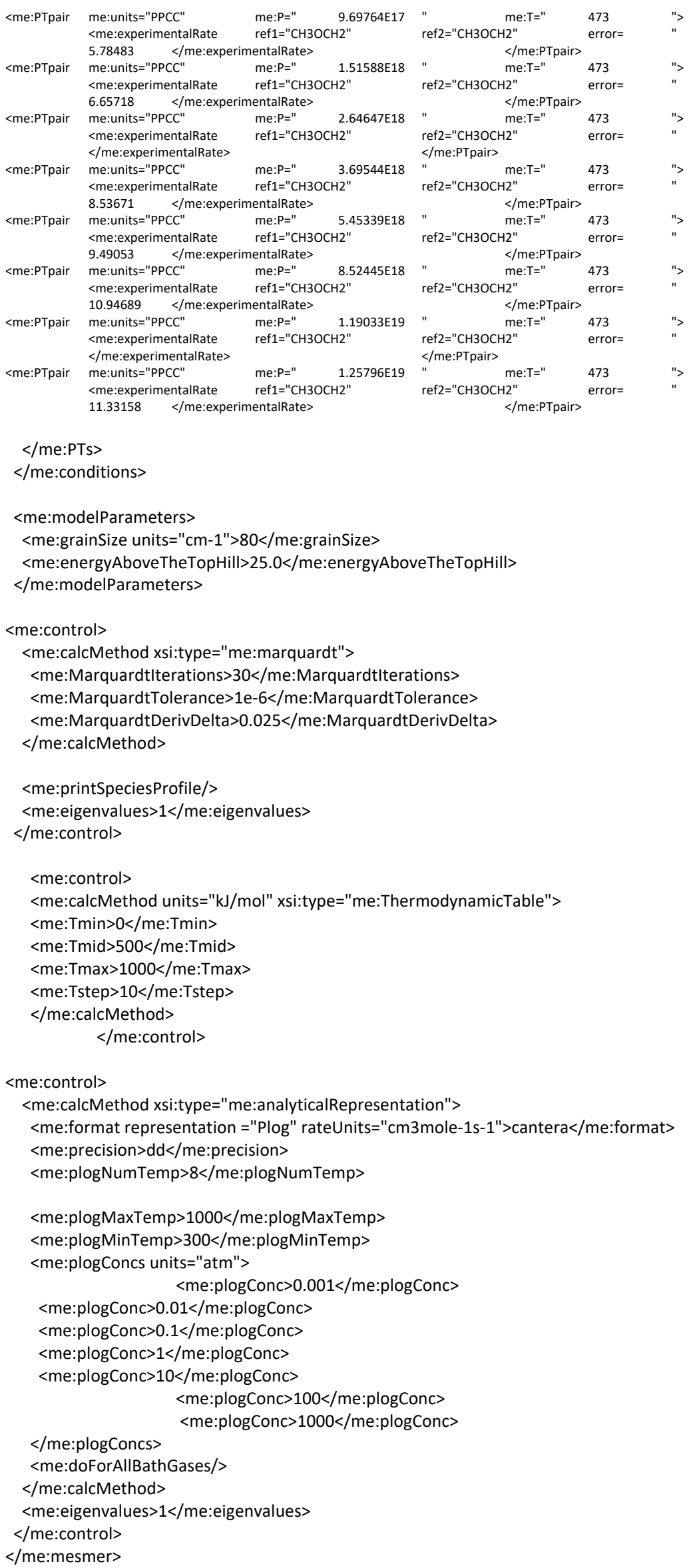

$</$ me:PTs $>$

$</$ me:conditions $>$

$<$ me:modelParameters $>$

$<$ me:grainSize units="cm-1">80</me:grainSize>

$<$ me:energyAboveTheTopHill>25.0</me:energyAboveTheTopHill>

$<$ me:modelParameters $>$

$<$ me:control>

$<$ me:calcMethod xsi:type="me:marquardt">

$<$ me:Marquardtlterations $>30</$ me:Marquardtlterations $>$

$<$ me:MarquardtTolerance $>1 \mathrm{e}-6<$ /me:MarquardtTolerance $>$

$<$ me:MarquardtDerivDelta $>0.025</$ me:MarquardtDerivDelta $>$

$<$ /me:calcMethod $>$

$<$ me:printSpeciesProfile/>

$<$ me:eigenvalues $>1</$ me:eigenvalues $>$

$<$ me:control>

$<$ me:control>

$<$ me:calcMethod units="kJ/mol" xsi:type="me:ThermodynamicTable">

$<$ me:Tmin $>0</$ me:Tmin $>$

$<$ me:Tmid $>500</$ me:Tmid $>$

$<$ me:Tmax $>1000<$ me:Tmax $>$

$<$ me:Tstep $>10<$ me:Tstep $>$

$</$ me:calcMethod $>$

$</$ me:control>

$<$ me:control>

$<$ me:calcMethod xsi:type="me:analyticalRepresentation">

$<$ me:format representation ="Plog" rateUnits="cm3mole-1s-1" >cantera</me:format $>$

$<$ me:precision $>$ dd $</$ me:precision $>$

$<$ me:plogNumTemp $>8</$ me:plogNumTemp $>$

<me:plogMaxTemp>1000</me:plogMaxTemp>

$<$ me:plogMinTemp>300</me:plogMinTemp>

$<$ me:plogConcs units="atm">

$<$ me:plogConc $>0.001</$ me:plogConc $>$

$<$ me:plogConc $>0.01</$ me:plogConc $>$

$<$ me:plogConc $>0.1</$ me:plogConc $>$

$<$ me:plogConc $>1</$ me:plogConc $>$

$<$ me:plogConc $>10</$ me:plogConc $>$

$<$ me:plogConc $>100<$ me:plogConc $>$

$</$ me:plogConcs $>$

$<$ me:plogConc $>1000</$ me:plogConc $>$

$<$ me:doForAllBathGases/>

$<$ /me:calcMethod $>$

$<$ me:eigenvalues $>1</$ me:eigenvalues $>$

$</$ me:control $>$

$</$ me:mesmer $>$ 